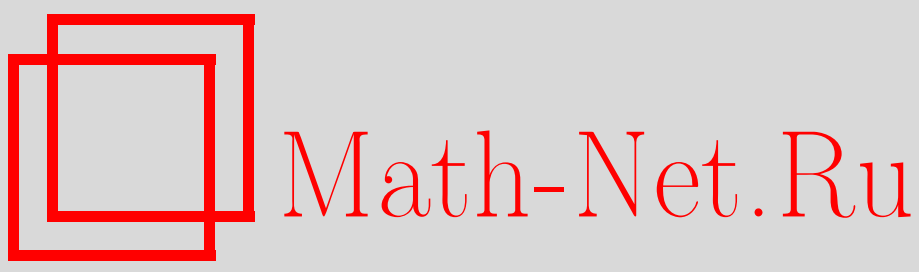

О. В. Матвеев, Интерполирование $D^{m}$-сплайнами и базисы в пространствах Соболева, Матем. сб., 1998, том 189, номер 11, 75-102

DOI: https://doi.org/10.4213/sm371

Использование Общероссийского математического портала Math-Net.Ru подразумевает, что вы прочитали и согласны с пользовательским соглашением

http://www . mathnet.ru/rus/agreement

Параметры загрузки:

IP: 54.196 .121 .252

26 апреля 2023 г., 13:59:13 
УДК 517.518

\section{О.В. Матвеев}

\section{Интерполирование $D^{m}$-сплайнами и базисы в пространствах Соболева}

Рассматривается приближение функций многих переменных интерполяционными $D^{m}$-сплайнами на нерегулярных сетках. Получены точные по порядку оценки (различных типов) погрешности приближения функций $f \in W_{p}^{k}(\Omega)$ в полунормах $\left\|D^{l} \cdot\right\|_{L_{q}}$ через модули гладкости $k$-х производных $f$ в $L_{p}(\Omega)$. Как следствие этих результатов для любого $t \in \mathbb{N}$ построен базис в пространстве Соболева $W_{p}^{k}(\Omega)$, где $\Omega$ - ограниченная область в $\mathbb{R}^{n}$ с минимально гладкой границей такой, что погрешность приближения функции $f \in W_{p}^{k}(\Omega) N$-й частичной суммой ее разложения по этому базису оценивается через модуль гладкости $\omega_{t}\left(D^{k} f, N^{-1 / n}\right)_{L_{p}(\Omega)}$ порядка $t$.

Библиограф̆ия: 44 названия.

\section{Введение}

Пусть $\Omega$ - область в $\mathbb{R}^{n}$ с минимально гладкой границей $[1], \Delta \subset \bar{\Omega}, m$ - целое, $m>n / 2$, и $L_{2}^{m}(\Omega)$ - множество непрерывных функций $g: \bar{\Omega} \rightarrow \mathbb{R}$, у которых все обобщенные производные порядка $m$ принадлежат $L_{2}(\Omega) .{ }^{1}$ Интерполяиионным $D^{m}$-сплайном $s=s(f, \Delta, m, \Omega)$ в простейшем случае называется решение вариационной задачи

$$
\min \left\{\int_{\Omega} \sum_{|\alpha|_{1}=m} \frac{m !}{\alpha !}\left(D^{\alpha} g\right)^{2} d x: g \in L_{2}^{m}(\Omega), g(x)=f(x) \forall x \in \Delta\right\},
$$

где $f$ - интерполируемая функция, определенная на $\bar{\Omega}, \alpha=\left(\alpha_{1}, \ldots, \alpha_{n}\right)$ - мультииндекс, $|\alpha|_{1}=\alpha_{1}+\cdots+\alpha_{n}, \alpha !=\alpha_{1} ! \cdots \alpha_{n}$ ! (см. [2]). Эта вариационная задача имеет решение, если ее допустимое множество непусто, и это решение единственно тогда и только тогда, когда не существует ненулевого полинома степени $<m$, равного нулю на $\Delta[3]$. Множество $\Delta$ здесь может быть дискретным (т.е. сеткой), но в обшем случае $\Delta$ - произвольное подмножество $\bar{\Omega}$. Наряду с термином “ $D^{m}$-сплайны” употребляются также и другие названия: “функции тонких пластин”, “полигармонические сплайны", “сплайны на хаотических сетках"; в дальнейшем мы будем говорить просто “сплайны”. Данньй метод интерполирования линеен и коммутирует с движениями пространства. Сплайн $s(x)$ - полигармоническая функция порядка $m$ на $\Omega \backslash \bar{\Delta}$, так как уравнением Эйлера для задачи (1)

Работа выполнена при финансовой поддержке Российского фонда фундаментальных исследований (грант № 98-01-00047).

${ }^{1}$ При этом сужение $g$ на любое ограниченное открытое множество $G \subset \Omega$ принадлежит пространству Соболева $W_{2}^{m}(G)$, см., например, [3; лемма 4$]$. 
является полигармоническое уравнение. В частности, при $n=1$ получаем обычные кусочно полиномиальные сплайны степени $2 m-1$ дефекта 1.

Через $W_{p}^{k}(G)$, где $G$ - открытое множество в $\mathbb{R}^{n}, k$ - целое неотрицательное, $1 \leqslant p \leqslant \infty$, будем обозначать пространство Соболева [4], видоизмененное следующим образом: при $p=\infty$ наряду с ограниченностью требуется равномерная непрерывность на $G$ производных порядка $\leqslant k$ функции $g \in W_{p}^{k}(G)$. Положим $\left\|D^{k} g\right\|_{L_{p}(G)}=\max _{|\alpha|_{1}=k}\left\|D^{\alpha} g\right\|_{L_{p}(G)}$,

$$
\bar{h}=\bar{h}(\Delta)=\sup _{x \in \Omega} \inf _{y \in \Delta}|x-y|, \quad \underline{h}=\underline{h}(\Delta)=\inf _{\substack{x, y \in \Delta \\ x \neq y}}|x-y|
$$

(инфимум по пустому множеству считаем равным $+\infty$ ).

Аппроксимативные свойства сплайнов исследовались в работах автора [3], [5], [6], в которых получены точные по порядку (относительно $\bar{h}$ ) оценки погрешности приближения вида

$$
\begin{aligned}
& \left\|D^{l}(f-s)\right\|_{L_{q}(\Omega)} \leqslant C \bar{h}^{\theta}\left\|D^{k} f\right\|_{L_{p}(\Omega)}, \\
& \left\|D^{l}(f-s)\right\|_{L_{q}(\Omega)} \leqslant C \bar{h}^{\theta} \omega\left(D^{k} f, \bar{h}\right)_{L_{p}(\Omega)},
\end{aligned}
$$

а также изучены свойства сходимости сплайнов к интерполируемой функции в пространствах $W_{p}^{k}(\Omega)$. Здесь $\omega$ - модуль непрерывности,

$$
\theta=\theta(k, p)= \begin{cases}k-l-\frac{n}{p}+\frac{n}{q} & \text { при } p \leqslant q, \\ k-l & \text { при } p>q,\end{cases}
$$

$C$ - константа, завислщая от $\Omega, m, p, q$ либо от $\Omega, m, p, q, \varkappa$, где $\varkappa$ - величина, удовлетворяющая неравенствам ${ }^{2}$

$$
\varkappa>0, \quad \underline{h}>\varkappa \bar{h} .
$$

В указанных работах эти вопросы исследованы для произвольных $\Delta, \Omega, m, n, k, l$, $p, q$, удовлетворяющих некоторым естественным условиям.

1) Случай $n=1$ (Дж. Алберг, Э. Нильсон, Дж. Уолш [7]-[11], А. Шарма, А. Меир [12], [13], С. Норд [14], К. де Бор [15], Ю.Н. Субботин [16]-[18], Т. Лукас [19], М. Марсден [20], Н. Л. Зматраков [21], А. Ю. Шадрин [22] и др.; см. также монографии [23]-[26]). В работе [22] получены неравенства (2), (3) при произвольных $\Delta, m, k, l, p, q$ (считается, что $\Omega$ - конечный интервал, но это ограничение несущественно); более того, получена оценка погрешности приближения через модуль гладкости порядка $m-k$ производной $f^{(k)}$ в правой части. В большинстве работ, посвященных сплайнам одного переменного, используется алгебраический подход: сплайн является кусочно полиномиальной функцией, причем коэффициенты соответствующих полиномов удовлетворяют некоторой системе линейных уравнений; используются свойства возникающих при этом матрищ (диагональное преобладание, цикличность, осцилляционные матрицы и др.). Кроме того, в [15], [18], [22]

\footnotetext{
${ }^{2}$ Имеется ввиду, что во втором случае $C=g(\bar{h} / \underline{h})$, где $g:[0,+\infty) \rightarrow \mathbb{R}-$ некоторая возрастающая неограниченная функция, зависящая от $\Omega, m, p, q, \underline{\text { и }} \underline{h}>0$.
} 
используется свойство экспоненциального убьвания фундаментального сплайна (в соответствующих нормах), однако в доказательствах этого свойства также существенную роль играет кусочно полиномиальная структура сплайна. В немногих случаях найдены точные мультипликативные константы в оценках погрешности сплайн-интерполяции [26].

Попутно отметим, что уже при $m=2, \Omega=(0,1)$ (кубические сплайны) обнаружился следующий факт: при некоторых $k, l, p, q$ для любого $f \in W_{p}^{k}(\Omega)$ сплайны $s\left(f, \Delta_{i}, m, \Omega\right)$ сходятся к $f$ в $W_{q}^{l}(\Omega)$, если $\Delta_{i} \subset \bar{\Omega}(i \in \mathbb{N})$ и $\lim _{i \rightarrow \infty} \bar{h}\left(\Delta_{i}\right)=0$, а при других $k, l, p, q$ это неверно, но сходимость имеет место, если наложить некоторое дополнительное условие на последовательность $\left\{\Delta_{i}\right\}$. В качестве такого условия часто берется условие квазиравномерности: $\bar{h}\left(\Delta_{i}\right)=O\left(\underline{h}\left(\Delta_{i}\right)\right),{ }^{3}$ хотя рассматривались и другие ограничения. Аналогично, в некоторых случаях оценка (2) или (3) выполняется только с константой $C$, зависяшей от $\varkappa$, где $\varkappa$ удовлетворяет (4).

2) $k=m, p=2, \Omega$ - ограниченная область. Здесь Ж. Дюшон [28], [29] и А. Ю. Бежаев [30] использовали подход, основанньй непосредственно на вариационном принципе. В [30] установлено неравенство (2) и доказана сходимость $s\left(f, \Delta_{i}, m, \Omega\right)$ к $f$ в $W_{2}^{m}(\Omega)$ для любого $f \in W_{2}^{m}(\Omega)$ при $\bar{h}\left(\Delta_{i}\right) \rightarrow 0$. В более ранних работах [28], [29] получены аналогичные результаты для сплайнов $s\left(f, \Delta, m, \mathbb{R}^{n}\right)$, т.е. функция $f$, определенная на $\Omega$, приближается сплайном, определенным на $\mathbb{R}^{n}$ (подробнее об этом сказано ниже).

3) $\Omega=\mathbb{R}^{n}, \Delta=h \mathbb{Z}^{n}(h>0)$. При этих условиях У. Мэдич и С. Нельсон [31], применяя методы гармонического анализа, доказали экспоненциальное убьвание фундаментального сплайна в норме $\|\cdot\|_{L_{\infty}}$ (здесь удается найти в явном виде преобразование Фурье фундаментального сплайна). Отсюда можно вывести оценки вида (2), (3).

В настоящей статье получены оценки погрешности приближения сплайнами через модули гладкости производных интерполируемой функции, в частности неравенство вида

$$
\left\|D^{l}(f-s)\right\|_{L_{q}(\Omega)} \leqslant C \bar{h}^{\theta} \omega_{m-k}\left(D^{k} f, \bar{h}\right)_{L_{p}(\Omega)}
$$

(где $\omega_{m-k}$ можно заменить на $\omega_{2 m-k}$ при $\Omega=\mathbb{R}^{n}$ ), которое является усилением установленных в [3], [5], [6] оценок $(2),(3)$. Мы пользуемся обозначениями

$$
\begin{aligned}
\omega_{t}\left(D^{k} g, \delta\right)_{L_{p}(G)} & =\max _{|\alpha|_{1}=k} \omega_{t}\left(D^{\alpha} g, \delta\right)_{L_{p}(G)}, \\
\omega_{t}(g, \delta)_{L_{p}(G)} & =\sup _{|a| \leqslant \delta}\left\|\sum_{i=0}^{t}(-1)^{i}\left(\begin{array}{c}
t \\
i
\end{array}\right) g(x+i a)\right\|_{L_{p}\left(G_{a}\right)},
\end{aligned}
$$

где $t$ - целое неотрицательное, $G_{a}$ - множество точек $x \in \mathbb{R}^{n}$ таких, что отрезок $[x, x+t a]$ содержится в $G$. Кроме того, получены оценки других типов в ситуациях, ког да оценка (5) оказывается недостаточной (приближение на компактных подмножествах $\Omega$, а также случай $k=m, \theta=0$ ). Рассматриваются и другие вопросы: точный порядок сходимости на соболевских классах интерполируемых функций, возможность аналитического представления сплайна.

\footnotetext{
3 Это условие появлялось ранее и в многомерном случае, например при исследовании метода интерполирования Шепарда [27].
} 
Простьм следствием неравенства (5) является один результат, касающийся базисов в пространствах Соболева. Базисы в пространствах $W_{p}^{k}(\Omega)$ (вложенных в $\left.W_{\infty}^{0}(\Omega)\right)$, где $\Omega$ - ограниченная область в $\mathbb{R}^{n}$ с минимально гладкой гранищей, были построены автором с помощью сплайнов $s(f, \Delta, m, \Omega)$ в работах [3], [6], в которых также фактически доказано неравенство

$$
\sum_{l=0}^{k}\left\|D^{l}\left(f-\sum_{i=1}^{N} \lambda_{i} u_{i}\right)\right\|_{L_{p}(\Omega)} N^{(k-l) / n} \leqslant C \omega_{t}\left(D^{k} f, N^{-1 / n}\right)_{L_{p}(\Omega)}
$$

при $t=1$ (для достаточно больших $N$ ), где $\sum_{i=1}^{\infty} \lambda_{i} u_{i}-$ разложение функции $f \in W_{p}^{k}(\Omega)$ по специальному базису $\left\{u_{i}\right\}, C=C(\Omega, k, p)$ (т.е. $C$ - некоторая константа, зависящая только от $\Omega, k, p)$. В данной работе оценка (6) установлена для тех же базисов при любом $t$. Точнее, для произвольных $t \in \mathbb{N}$ и $\Omega$ (здесь $\Omega$ ограничено) строится система функций $\left\{u_{i}: i \in \mathbb{N}\right\} \subset W_{\infty}^{t}(\Omega)$, являющаяся базисом в каждом пространстве $W_{p}^{k}(\Omega)$, где $k \leqslant t$ и $W_{p}^{k}(\Omega)$ вложено в $W_{\infty}^{0}(\Omega)$, причем выполняется (6) с константой $C=C(\Omega, t, p)$. Ранее базисы в $W_{p}^{k}(\Omega)$ были найдены в случае, когда граница $\Omega$ имеет гладкость $C^{k}$ либо "склеена" из конечного числа частей, имеющих такую гладкость (З. Чисельский, Т. Фигель [32]). Отметим, что базисы в пространствах Соболева интенсивно изучались с конща 60-х годов (З. Чисельский, Ж. Домста, С. Шонфилд, Ю.Н. Субботин, С. Ропела, Т. Фигель и др.; см. библиографию в [32], а также цитированные здесь статьи). В ряде работ построены базисы, обладающие свойством (6). Вопрос о существовании для любого $t$ базиса в пространстве $C[0,2 \pi]$ непрерывных $2 \pi$-периодических функций со свойством (6), где $n=1, k=0, p=\infty, C=C(t)$, поставленный П.Л. Ульяновым, был решен Ю.Н. Субботиным, указавшим такие базисы [33]. Базис в $W_{p}^{k}(\Omega)$, где $\Omega$ - ограниченная область в $\mathbb{R}^{n}$ с границей, состоящей из конечного числа частей гладкости $C^{k}$, для которого вьполняется (6) (где $C=C(\Omega, k, t), t$ - произвольное заданное), может быть построен с помощью результатов [34] (частное сообщение 3. Чисельского).

Методы, которые применялись ранее в задачах аппроксимации сплайнами в частных случаях, опираются на специфику этих случаев и поэтому не могут быть применены в общей ситуации. Аппарат, используемьй в данной работе, включает три основных момента: 1) ряд фактов теории функций многих действительных переменных - леммы 1-5, 12 (а также теоремы вложения и теоремы продолжения для пространств Соболева); 2) свойство экспоненциального убывания сплайна на подобласти $\Omega$, содержашей нули интерполируемой функции (установленное в общем виде автором в [3]); 3) неравенство типа Маркова-Бернштейна-Никольского для сплайнов (лемма 9).

Заканчивая вводную часть статьи, остановимся на вопросе об аналитической структуре сплайна. Если $\Omega=\mathbb{R}^{n}$ и $\Delta$ конечно, то сплайн $s(x)$ имеет вид

$$
\sum_{y \in \Delta} \xi_{y} K(x-y)+P(x)
$$

г де $K(x)=|x|^{2 m-n}$ при нечетном $n$ и $K(x)=|x|^{2 m-n} \ln |x|$ при четном $n, \xi_{y} \in \mathbb{R}$, $P$ - полином степени $<m$ (Ж. Дюшон [28]). При $n>1, \Omega \neq \mathbb{R}^{n}$, к сожалению, неизвестны какие-либо подобные представления сплайна в явном виде (при $n>1$ 
в отличие от случая $n=1$ сплайн зависит от области, т.е. если $\Omega \subset \Omega^{\prime}$, то сужение $s\left(f, \Delta, m, \Omega^{\prime}\right)$ на $\Omega$, вообше говоря, не совпадает с $\left.s(f, \Delta, m, \Omega)\right)$. Однако для приближения функции $f$ на области $\Omega$ можно использовать сплайн $s\left(f, \Delta, m, \mathbb{R}^{n}\right)$, причем такой метод приближенного восстановления функций по их сеточным значениям показал хорошие результаты в численных экспериментах, проводившихся при $m=n=2$ (см. [35]-[37]). Так, в сравнительных тестах, проведенных Р. Франке [35] для большой групшы разнообразных методов, $D^{m}$-сплайны дали наилучшую точность приближения. В некоторых случаях, правда, аппроксимативные свойства сплайнов $s\left(f, \Delta, m, \mathbb{R}^{n}\right)$ оказываются хуже, чем свойства сплайнов $s(f, \Delta, m, \Omega)$ [6; замечание 6].

В настоящей работе получены также оценки погрешности приближения (на ограниченной области $\Omega)$ сплайнами $s\left(f, \Delta, m, \mathbb{R}^{n}\right)$.

\section{§1. Оценки погрешности сплайн-интерполяции на всей области $\Omega$}

Прежде всего перечислим обозначения, которые будут использоваться на протяжении всей работы. $\mathrm{K}$ ним относятся уже введенные обозначения $m$, $n, \Omega, \Delta, \bar{h}=\bar{h}(\Delta), \underline{h}=\underline{h}(\Delta),|\alpha|_{1}, \alpha !, W_{p}^{k}(G),\left\|D^{k} g\right\|_{L_{p}(G)}, \omega_{t}(g, \delta)_{L_{p}(G)}$, $\omega_{t}\left(D^{k} g, \delta\right)_{L_{p}(G)}$ (в последних двух выражениях при $t=1$ будем опускать индекс $t), \theta=\theta(k, p), K(x)$. Далее, $\xi_{+}=\max \{\xi, 0\}$, где $\xi \in \mathbb{R} ; \mathbb{Z}_{+}=\mathbb{N} \cup\{0\}$; $x^{\alpha}=x_{1}^{\alpha_{1}} \cdots x_{n}^{\alpha_{n}}$, где $x=\left(x_{1}, \ldots, x_{n}\right) \in \mathbb{R}^{n}, \alpha=\left(\alpha_{1}, \ldots, \alpha_{n}\right) \in \mathbb{Z}_{+}^{n}$ (считаем $\left.0^{0}=1\right) ; \mathscr{P}_{t}-$ множество полиномов от $n$ переменных степени $\leqslant t$ (по совокупности переменных), где $t \in \mathbb{Z}_{+} ; \mathscr{P}_{-1}=\{o\}$, где $o-$ нулевой полином; $B(a, r)=\left\{x \in \mathbb{R}^{n}:|x-a|<r\right\}, \operatorname{dist}(U, V)=\inf \{|x-y|: x \in U, y \in V\}$, $\operatorname{dist}(a, U)=\operatorname{dist}(\{a\}, U), \operatorname{diam} U=\sup \{|x-y|: x, y \in U\}, U_{(r)}=\left\{x \in \mathbb{R}^{n}\right.$ : $\overline{B(x, r)} \subset U\}, U^{(r)}=U \backslash \overline{U_{(r)}}$, где $a \in \mathbb{R}^{n}, r \in \mathbb{R}, U, V \subset \mathbb{R}^{n} ; \mathfrak{v}$ - объем шара $B(0,1) ; \Pi_{a \lambda}(r)$ - связная компонента множества $(\lambda \Omega) \cap B(a, r)$, содержащая $a$ при $r>0, \Pi_{F \lambda}(r)=\bigcup_{a \in F} \Pi_{a \lambda}(r), \Sigma_{F \lambda}(r)=(\lambda \Omega) \backslash \overline{\Pi_{F \lambda}(r)}$, где $\lambda>0, r \in \mathbb{R}, a \in \lambda \Omega$, $F \subset \lambda \Omega ; \mathscr{D}(G)$ - множество бесконечно дифференцируемых функций $g: \mathbb{R}^{n} \rightarrow \mathbb{R}$, имеющих компактные в $G$ носители, $W_{p}^{k}=W_{p}^{k}(B(0,1)), W_{p}^{k}(G, \mathrm{fin})$-пространство (классов) функций $g: G \rightarrow \mathbb{R}$, принадлежаших $W_{p}^{k}(G \cap B(0, r))$ при любом $r>0$, с топологией, порожденной семейством полунорм $\left\{\|g\|_{W_{p}^{k}(G \cap B(0, r))}: r>0\right\}, L_{p}^{k}(G)$ - подмножество $W_{p}^{k}(G, f i n)$, состоящее из функций, у которых все производные порядка $k$ принадлежат $L_{p}(G)$, где $G$ - открытое множество в $\mathbb{R}^{n}, k \in \mathbb{Z}_{+}$, $1 \leqslant p \leqslant \infty ; M_{t}$ - некоторое фиксированное подмножество $B\left(0, \frac{1}{2}\right)$ такое, что для любой функции $g: M_{t} \rightarrow \mathbb{R}$ сушествует единственньй полином из $\mathscr{P}_{t-1}$, интерполирующий ее на $M_{t}$ (можно взять, например, $M_{t}=\frac{1}{2 t}\left\{a \in \mathbb{Z}_{+}^{n}:|a|_{1}<t\right\}$ при $t>0$ и $M_{t}=\varnothing$ при $\left.t=0\right), P_{t}(g, y, \cdot)$ - полином из $\mathscr{P}_{t-1}$, интерполирующий функцию $g$ на множестве $y+M_{t}, H_{x y}^{t}$ - выпуклая оболочка множества $\{x\} \cup\{y\} \cup\left(y+M_{t}\right)$, где $t \in \mathbb{Z}_{+}, x, y \in \mathbb{R}^{n} ; X_{t}(G, \delta)$ - множество точек $(x, y) \in \mathbb{R}^{2 n}$ таких, что $H_{x y}^{t} \subset G \cap B(x, \delta)$,

$$
\begin{gathered}
\widetilde{\omega}_{t}(g, \delta)_{p, G}=\left\|g(x)-P_{t}(g, y, x)\right\|_{L_{p}\left(X_{t}(G, \delta)\right) \quad\left(g \in L_{p}(G)\right)} \\
\widetilde{\omega}_{t}\left(D^{k} g, \delta\right)_{p, G}=\max _{|\alpha|_{1}=k} \widetilde{\omega}_{t}\left(D^{\alpha} g, \delta\right)_{p, G} \quad\left(g \in L_{p}^{k}(G)\right)
\end{gathered}
$$

где $t \in \mathbb{Z}_{+}, \delta>0, G$ - открытое множество в $\mathbb{R}^{n} ; \varphi$ - некоторая фиксированная функция из $\mathscr{D}(B(0,1))$ такая, что $\varphi(x) \geqslant 0 \forall x \in \mathbb{R}^{n}, \varphi(x)=1 \forall x \in B\left(0, \frac{1}{3}\right)$, 


$$
\begin{aligned}
& \int_{\mathbb{R}^{n}} \varphi d x=1 \text { и } \\
& \varphi_{y}(x)=\varphi(x-y)\left(\sum_{z \in \frac{1}{2 n} \mathbb{Z}^{n}} \varphi(x-z)\right)^{-1},
\end{aligned}
$$

где $y \in \mathbb{R}^{n} ; \mathscr{L}$ - оператор Лапласа; $E_{1} \rightarrow E_{2}$ - непрерывное вложение $E_{1}$ в $E_{2}$, где $E_{1}, E_{2}$ - линейные топологические пространства.

Лемма 1. Пусть $\lambda \geqslant 1, a \in \mathbb{R}^{n}, V=(\lambda \Omega) \cap B(a, 1), V^{\prime}=(\lambda \Omega) \cap B(a, 3)$, $W_{p}^{k} \rightarrow W_{q}^{l}$ u $g \in W_{p}^{k}\left(V^{\prime}\right)$. Тогда $g \in W_{q}^{l}(V) u$

$$
\|g\|_{W_{q}^{l}(V)} \leqslant C\|g\|_{W_{p}^{k}\left(V^{\prime}\right)}
$$

әде $C=C(\Omega, k, p, q)$.

ДокАЗАтЕльство. Минимальная гладкость границы $\Omega$ означает наличие таких $\varepsilon>0$ и $L$, что для любой точки $b \in \mathbb{R}^{n}$ существуют функция $\psi_{b}: \mathbb{R}^{n-1} \rightarrow \mathbb{R}$ и движение $\Gamma_{b}: \mathbb{R}^{n} \rightarrow \mathbb{R}^{n}$, удовлетворяющие условиям: $\left|\psi_{b}(\xi)-\psi_{b}(\eta)\right| \leqslant L|\xi-\eta|$ $\forall \xi, \eta \in \mathbb{R}^{n-1}$ и $\Omega \cap B(b, \varepsilon)=\Gamma_{b}\left(\Lambda_{b}\right) \cap B(b, \varepsilon)$, где $\Lambda_{b}=\left\{x=\left(x_{1}, \ldots, x_{n}\right) \in \mathbb{R}^{n}:\right.$ $\left.x_{n}>\psi_{b}\left(x_{1}, \ldots, x_{n-1}\right)\right\}$ [1]. Очевидно, достаточно доказать лемму при $\lambda>3 / \varepsilon$, $p<\infty$. Тогда, полагая $b=\lambda^{-1} a, \Lambda=\lambda \Gamma_{b}\left(\Lambda_{b}\right)$, имеем

$$
(\lambda \Omega) \cap B(a, 3)=\Lambda \cap B(a, 3) .
$$

Согласно [1] функцию $g_{1}(x)$, равную $g(x) \varphi\left(\frac{x-a}{3}\right)$ при $x \in \Lambda \cap B(a, 3)$ и нулю при $x \in \Lambda \backslash B(a, 3)$ (совпадаюшую с $g(x)$ на $V$ ), можно продолжить на $\mathbb{R}^{n}$ так, чтобы $g_{1} \in W_{p}^{k}\left(\mathbb{R}^{n}\right)$ и $\left\|g_{1}\right\|_{W_{p}^{k}\left(\mathbb{R}^{n}\right)} \leqslant A\left\|g_{1}\right\|_{W_{p}^{k}(\Lambda)}$, где $A=A(n, L, k)$. Отсюда и из (8) следует утверждение леммы.

Всюду в работе предполагается, что $f \in W_{p}^{k}(\Omega, \mathrm{fin}), W_{p}^{k} \rightarrow W_{\infty}^{0}, W_{p}^{k} \rightarrow W_{q}^{l}$, множество $\Omega$ ограничено при $p>q ;^{4} \bar{h}<h^{*}$, где $h^{*}=h^{*}(\Omega, m)$ - положительная константа, которая будет определена ниже. Под шаром понимается непустой открытьй шар в $\mathbb{R}^{n}$ (т.е. множество $B(a, r)$ при некоторых $a \in \mathbb{R}^{n}, r>0$ ). Функции из $W_{\infty}^{0}(G, \mathrm{fin})$ мы будем считать определенными и непрерьвными на $\bar{G}$. Заметим, что по лемме 1 (в которой надо сделать очевидные замены параметров $k, l, p, q$ ) имеем $W_{p}^{k}(\Omega$, fin $) \rightarrow W_{\infty}^{0}(\Omega$, fin $), W_{2}^{m}(\Omega$, fin $) \rightarrow W_{\infty}^{0}(\Omega$, fin $)\left(W_{2}^{m} \rightarrow W_{\infty}^{0}\right.$, так как $m>n / 2[38 ; \S \S 9,10])$ и что минимальная гладкость границы области эквивалентна сильному условию конуса в смысле [38].

Во введении отмечалось, что сплайн $s(f, \Delta, m, \Omega)$, определенный как решение вариационной задачи, сушествует и единствен, если

1 ) сушествует функция из $L_{2}^{m}(\Omega)$, совпадаюшая с $f$ на $\Delta$,

2) $\left(P \in \mathscr{P}_{m-1}, P(x)=0 \forall x \in \Delta\right) \Rightarrow(P(x) \equiv 0)$.

Второе условие будет выполнено за счет того, что $\bar{h}<h^{*}$. Рассмотрим условие 1) и допустим сначала, что $\Omega$ ограничено. Ясно, что для выполнения этого условия необходимо наложить некоторые ограничения на $\Delta, k, p$. На протяжении всей работы будем считать, что либо $\underline{h}>0$, либо $W_{p}^{k} \rightarrow W_{2}^{m}$. Если же $\Omega$ неограничено, то условие 1), вообше говоря, не выполняется, даже если $\underline{h}>0$ и функция $f$ достаточно гладкая. Следовательно, нужна другая конструкция, более общая, чем (1).

\footnotetext{
${ }^{4}$ В противном случае, как нетрудно проверить [3], оценка (2) не имеет места.
} 
ОпРЕДЕЛЕниЕ [3; определение 2]. Функцию $s=s(f, \Delta, m, \Omega), s \in W_{2}^{m}(\Omega$, fin), будем называть интерполяиионным сплайном, если выполняются условия:

1) для любой функции $g \in W_{2}^{m}(\Omega)$, имеющей ограниченный носитель и равной нулю на $\Delta$,

$$
\int_{\Omega} \sum_{|\alpha|_{1}=m} \frac{m !}{\alpha !} D^{\alpha} s D^{\alpha} g d x=0
$$

2) $s(x)=f(x)$ на $\Delta$

3) если $a \in \Omega$ и $\zeta>1$, то $\|s\|_{W_{2}^{m}\left(\Pi_{a 1}(r)\right)}=O\left(\zeta^{r}\right)$ при $r \rightarrow \infty$.

Как показано в [3; теоремы 1,4$]$, в случае $f \in L_{p}^{k}(\Omega)$ определенньй таким образом сплайн $s$ сушествует и единствен. Если сушествует решение задачи (1), то оно удовлетворяет и условиям данного сейчас определения, так как свойством 3 ) обладает любая функция $s \in L_{2}^{m}(\Omega)[3 ;$ лемма 4$]$.

Теорема 1. Допустим, ито $f \in L_{p}^{k}(\Omega), W_{2}^{m} \rightarrow W_{q}^{l}$ и вылполняется 1) условие (4) или 2) $W_{p}^{k} \rightarrow W_{2}^{m}$. Тогда справедливы неравенства: при $k \leqslant m$

$$
\left\|D^{l}(f-s)\right\|_{L_{q}(\Omega)} \leqslant C \bar{h}^{\theta} \omega_{m-k}\left(D^{k} f, \bar{h}\right)_{L_{p}(\Omega)}
$$

u npu $k \leqslant 2 m, \Omega=\mathbb{R}^{n}$

$$
\left\|D^{l}(f-s)\right\|_{L_{q}(\Omega)} \leqslant C \bar{h}^{\theta} \omega_{2 m-k}\left(D^{k} f, \bar{h}\right)_{L_{p}(\Omega)},
$$

әде $C=C(\Omega, m, p, q, \varkappa)$ в случае 1$)$ и $C=C(\Omega, m, p, q)$ в случае 2$).^{5}$

Нам понадобится ряд вспомогательных утверждений.

Лемма 2 [3; лемма 1]. Для любого $\varepsilon \in\left(0, C_{1}\right]$ существуют области $G_{j} \subset \Omega$, $j \in J$, удовлетворяющие условиям:

1) $\operatorname{diam} G_{j} \leqslant C_{2} \varepsilon$

2) область $G_{j}$ звездна относительно некоторого шара радиуса $\varepsilon$ (т.е. звездна относительно каждой точки этого шара);

3) для любого $x \in \mathbb{R}^{n}$ найдется такое $j \in J$, ито $\Omega \cap B(x, \varepsilon) \subset G_{j}$;

4) любой шар радиуса в пересекает не более чем $C_{3}$ множеств $G_{j}$. Здесь $C_{i}=C_{i}(\Omega)>0$.

ЛЕмма 3. Пусть $G$ - ограниченная область, звездная относительно шара $B(0,1), \operatorname{diam} G \leqslant d, g \in W_{p}^{k}(G), t \in \mathbb{Z}_{+}, t \geqslant k$. Тогда существует полином $P \in \mathscr{P}_{t-1}$, для которого

$$
\|g-P\|_{W_{p}^{k}(G)} \leqslant C \widetilde{\omega}_{t-k}\left(D^{k} g, d\right)_{p, G},
$$

əде $C=C(n, d, t)$.

\footnotetext{
${ }^{5}$ Когда это не вызывает недоразумений, разные константы мы обозначаем одинаковыми буквами $C, C_{i}$.
} 
ДоказАТЕЛЬство. Определим операторы $P_{t}, Q_{t}: L_{1}(G) \rightarrow \mathscr{P}_{t-1}$ следуюшим образом:

$$
\begin{aligned}
P_{t}(u)(x) & =\frac{1}{\mathfrak{v}_{1}} \int_{B\left(0, \frac{1}{2}\right)} P_{t}(u, y, x) d y \\
Q_{t}(u)(x) & =\sum_{|\alpha|_{1}<t} \frac{(-1)^{|\alpha|_{1}}}{\alpha !} \int_{B(0,1)} u(y) D_{y}^{\alpha}\left(\varphi(y)(x-y)^{\alpha}\right) d y,
\end{aligned}
$$

где $\mathfrak{v}_{1}=2^{-n_{\mathfrak{v}}}$, а $D_{y}^{\alpha}$ обозначает дифференцирование по $y$. При $u \in L_{p}(G)$

$$
\begin{aligned}
\left\|u-P_{t}(u)\right\|_{L_{p}(G)} & =\frac{1}{\mathfrak{v}_{1}}\left\|\int_{B\left(0, \frac{1}{2}\right)}\left(u(x)-P_{t}(u, y, x)\right) d y\right\|_{L_{p}(G)} \\
& \leqslant \frac{1}{\mathfrak{v}_{1}} \int_{B\left(0, \frac{1}{2}\right)}\left\|u(x)-P_{t}(u, y, x)\right\|_{L_{p}(G)} d y \\
& \leqslant \mathfrak{v}_{1}^{-1 / p} \widetilde{\omega}_{t}(u, d)_{p, G} .
\end{aligned}
$$

Теперь предположим, что $u$ имеет обобшенную производную $D^{\beta} u \in L_{1}(G)$, где $|\beta|_{1} \leqslant t$. Тогда

$$
\begin{aligned}
D^{\beta} Q_{t}(u)(x) & =\sum_{\substack{|\alpha|_{1}<t \\
\alpha \geqslant \beta}} \frac{(-1)^{|\alpha|_{1}}}{(\alpha-\beta) !} \int_{B(0,1)} u(y) D_{y}^{\alpha}\left(\varphi(y)(x-y)^{\alpha-\beta}\right) d y, \\
Q_{t-|\beta|_{1}}\left(D^{\beta} u\right)(x) & =\sum_{|\alpha|_{1}+|\beta|_{1}<t} \frac{(-1)^{|\alpha|_{1}+|\beta|_{1}}}{\alpha !} \int_{B(0,1)} u(y) D_{y}^{\alpha+\beta}\left(\varphi(y)(x-y)^{\alpha}\right) d y
\end{aligned}
$$

( $\alpha \geqslant \beta$ означает, что $i$-я компонента $\beta$ не превосходит $i$-й компоненты $\alpha, i=\overline{1, n}$ ), поэтому

$$
D^{\beta} Q_{t}(u)=Q_{t-|\beta|_{1}}\left(D^{\beta} u\right)
$$

Кроме того, если в (11) проинтегрировать по частям и учесть формулу Тейлора, получим

$$
Q_{t}(u)=u \quad \forall u \in \mathscr{P}_{t-1} .
$$

Ниже $A_{i}=A_{i}(n, d, t)$. В силу (12)-(14) при $|\beta|_{1}=k$ имеем

$$
\begin{aligned}
\left\|D^{\beta}\left(g-Q_{t}(g)\right)\right\|_{L_{p}(G)}= & \left\|D^{\beta} g-Q_{t-k}\left(D^{\beta} g\right)\right\|_{L_{p}(G)} \\
\leqslant & \left\|D^{\beta} g-P_{t-k}\left(D^{\beta} g\right)\right\|_{L_{p}(G)} \\
& +\left\|Q_{t-k}\left(D^{\beta} g-P_{t-k}\left(D^{\beta} g\right)\right)\right\|_{L_{p}(G)} \\
\leqslant & A_{1} \widetilde{\omega}_{t-k}\left(D^{\beta} g, d\right)_{p, G} .
\end{aligned}
$$

Для любой функции $u \in W_{p}^{k}(G)$ сушествует такой полином $R(u) \in \mathscr{P}_{k-1}$, что

$$
\|u-R(u)\|_{W_{p}^{k}(G)} \leqslant A_{2}\left\|D^{k} u\right\|_{L_{p}(G)}
$$

(см., например, [3; предложение 1]). Полагая $P=Q_{t}(g)+R\left(g-Q_{t}(g)\right)$, в силу (15) и (16) наконец получаем

$$
\|g-P\|_{W_{p}^{k}(G)} \leqslant A_{2}\left\|D^{k}\left(g-Q_{t}(g)\right)\right\|_{L_{p}(G)} \leqslant A_{1} A_{2} \widetilde{\omega}_{t-k}\left(D^{k} g, d\right)_{p, G} .
$$


Лемма 4. Пусть $\lambda \geqslant C_{1}, a \in \lambda \Omega, g \in W_{p}^{k}\left(\lambda \Omega\right.$, fin), $t \in \mathbb{Z}_{+}, t \geqslant k$. Тогда существует такой полином $P \in \mathscr{P}_{t-1}$, что для любого $r \geqslant 1$

$$
\|g-P\|_{W_{p}^{k}\left(\Pi_{a \lambda}(r)\right)} \leqslant C_{2} r^{C_{3}} \widetilde{\omega}_{t-k}\left(D^{k} g, C_{4}\right)_{p, \Pi_{a \lambda}\left(r+C_{5}\right)} .
$$

Здесъ $C_{i}=C_{i}(\Omega, t)$.

ДокаЗАТЕЛЬство. Обозначим константы $C_{1}, C_{2}$ из леммы 2 через $\varepsilon_{0}, \rho$ соответственно и будем считать, что $\lambda \geqslant \rho / \varepsilon_{0}$. Пусть $G_{j}, j \in J,-$ области, удовлетворяюшие условиям 1$)-4)$ из леммы 2 при $\varepsilon=\rho / \lambda, G_{j}^{\prime}=\lambda G_{j}$. При некотором $j_{0}$ точка $a \in G_{j_{0}}^{\prime}$. Зафиксируем $r \geqslant 1$ и возьмем какое-либо $e \in J$ такое, что $G_{e}^{\prime} \cap$ $\Pi_{a \lambda}(r) \neq \varnothing$. Далее $A_{i}=A_{i}(\Omega, t)$. Выберем путь $\pi:[0,1] \rightarrow \Pi_{a \lambda}(r)$, для которого $\pi(0)=a, \pi(1) \in G_{e}^{\prime}$. Построим по индукции последовательность $j_{0}, j_{1}, \ldots, j_{c} \in J$. Если уже определено $j_{i}$, положим $\zeta_{i}=\sup \left\{\zeta \in[0,1]: \pi(\zeta) \in G_{j_{i}}^{\prime}\right\}$ и в случае $\zeta_{i}<1$ найдем такое $j_{i+1}$, что $\pi\left(\zeta_{i}\right) \in G_{j_{i+1}}^{\prime}$. Поскольку $\zeta_{i}<\zeta_{i+1}$ и количество областей $G_{j}^{\prime}$, пересекающих $\Pi_{a \lambda}(r)$, не превосходит $A_{1} r^{n}$, для некоторого $c-1 \leqslant A_{1} r^{n}$ получим $\zeta_{c-1}=1$. Наконец, $j_{c}=e$. Заметим, что любые две области $G_{j_{i}}, G_{j_{i+1}}$ пересекаются, поэтому согласно [3; замечание 1$]$ при подходяшем $j_{i}^{\prime}$ каждое из множеств $G_{j_{i}} \cap G_{j_{i}^{\prime}}, G_{j_{i+1}} \cap G_{j_{i}^{\prime}}$ содержит шар радиуса $\varepsilon / \rho$. Положим $U_{2 i}=G_{j_{i}}^{\prime}(i=\overline{0, c})$, $U_{2 i+1}=G_{j_{i}^{\prime}}^{\prime}(i=\overline{0, c-1})$. Тогда

$1)$ каждая из областей $U_{0}, \ldots, U_{2 c}$ имеет диаметр $\leqslant A_{2}$ и звездна относительно некоторого шара радиуса 1 ;

2) $U_{i} \subset \Pi_{a \lambda}\left(r+A_{3}\right)(i=\overline{0,2 c})$;

3) $B_{i} \subset U_{i} \cap U_{i+1}(i=\overline{0,2 c-1})$, где $B_{i}-$ некоторьй шар радиуса 1 ;

4) $U_{0}=G_{j_{0}}^{\prime}, U_{2 c}=G_{e}^{\prime}$;

5) количество этих областей $2 c+1 \leqslant A_{4} r^{n}$.

Пусть $P_{i}$ - полином из $\mathscr{P}_{t-1}$, удовлетворяющий неравенству

$$
\left\|g-P_{i}\right\|_{W_{p}^{k}\left(U_{i}\right)} \leqslant A_{5} \widetilde{\omega}_{t-k}\left(D^{k} g, A_{2}\right)_{p, U_{i}}
$$

(лемма 3). Имеем

$$
\begin{aligned}
\left\|P_{0}-P_{2 c}\right\|_{W_{p}^{k}\left(U_{2 c}\right)} & \leqslant \sum_{i=0}^{2 c-1}\left\|P_{i}-P_{i+1}\right\|_{W_{p}^{k}\left(U_{2 c}\right)} \\
& \leqslant \sum_{i=0}^{2 c-1} A_{6} r^{A_{7}}\left\|P_{i}-P_{i+1}\right\|_{W_{p}^{k}\left(B_{i}\right)} \\
& \leqslant \sum_{i=0}^{2 c-1} A_{6} r^{A_{7}}\left(\left\|g-P_{i}\right\|_{W_{p}^{k}\left(U_{i}\right)}+\left\|g-P_{i+1}\right\|_{W_{p}^{k}\left(U_{i+1}\right)}\right) \\
& \leqslant A_{8} r^{A_{9}} \widetilde{\omega}_{t-k}\left(D^{k} g, A_{2}\right)_{p, \Pi_{a \lambda}\left(r+A_{3}\right)} .
\end{aligned}
$$

Из (18) при $i=2 c$ и (19) следует

$$
\left\|g-P_{0}\right\|_{W_{p}^{k}\left(G_{e}^{\prime}\right)} \leqslant A_{10} r^{A_{9}} \widetilde{\omega}_{t-k}\left(D^{k} g, A_{2}\right)_{p, \Pi_{a \lambda}\left(r+A_{3}\right)} .
$$

Положим $P=P_{0}\left(P_{0}\right.$ не зависит от $\left.r, e\right)$. Для получения $(17)$ остается просуммировать оценки (20) по всем $e \in J$ таким, что $G_{e}^{\prime} \cap \Pi_{a \lambda}(r) \neq \varnothing$, и учесть, что количество таких $e$ не превосходит $A_{1} r^{n}$.

Отметим, что в леммах 3,4 условие $W_{p}^{k} \rightarrow W_{\infty}^{0}$ не используется. 
Лемма 5. Если $t \in \mathbb{Z}_{+}, \delta>0, G$ - открытое множество в $\mathbb{R}^{n}, g \in L_{p}(G)$ и при $p=\infty$, кроме того, $g \in W_{\infty}^{0}(G, \mathrm{fin})$, то $\widetilde{\omega}_{t}(g, \delta)_{p, G} \leqslant C \omega_{t}(g, \delta)_{L_{p}(G)}$, где $C=C(n, t, \delta)$.

ДокаЗАтеЛЬСтво. Пусть $z \in \mathbb{R}^{n}$ и $H_{0 z}^{t} \subset B(0, \delta)$. Ниже $A_{i}=A_{i}(n, t, \delta)$. Нам понадобятся следующие обозначения: $P_{a}$ - полином степени $<t$, равный 1 в точке $a \in M_{t}$ и нулю в остальных точках $M_{t}$;

$$
\tau(u)=u(0)-\sum_{a \in M_{t}} P_{a}(-z) u(a+z),
$$

где $u \in C\left(H_{0 z}^{t}\right) ; F$ - множество точек $(\xi, \eta) \in \mathbb{R}^{2 n}$ таких, что $\xi, \xi+t \eta \in H_{0 z}^{t} ; E-$ подпространство $C(F)$, состояшее из функций $v$, которые могут быть представлены в виде

$$
v(\xi, \eta)=\sum_{i=0}^{t}(-1)^{i}\left(\begin{array}{l}
t \\
i
\end{array}\right) u(\xi+i \eta),
$$

где $u$ - некоторая функция из $C\left(H_{0 z}^{t}\right)$. Допустим, что $u$ и $v$ связаны соотношением (21). Заметим, что, во-первых, как легко проверить, $\tau(P)=0$ для любого $P \in \mathscr{P}_{t-1}$ и, во-вторых, согласно [39]

$$
\inf _{P \in \mathscr{P}_{t-1}}\|u-P\|_{C\left(H_{0 z}^{t}\right)} \leqslant A_{1}\|v\|_{C(F)} .
$$

Поэтому $|\tau(u)| \leqslant A_{2}\|v\|_{C(F)}$. Отсюда следует, что $\tau(u)$ принимает одно и то же значение для всех $u$, удовлетворяющих (21) при фиксированном $v$, и что норма линейного функционала $v \rightarrow \tau(u)$, определенного на пространстве $E$, не превосходит $A_{2}$. По теореме Хана-Банаха этот функционал может быть продолжен на пространство $C(F)$ с той же нормой, и если использовать аналитическое представление ограниченных линейных функционалов в $C$ (см. [40]), то получится, что

$$
\tau(u)=\int_{F} \sum_{i=0}^{t}(-1)^{i}\left(\begin{array}{l}
t \\
i
\end{array}\right) u(\xi+i \eta) d \psi
$$

где $\psi$ - некоторая счетноаддитивная функция, определенная на борелевских подмножествах $F$, с вариацией $\|\psi\| \leqslant A_{2}$.

Теперь допустим, что $g \in W_{\infty}^{0}(G$, fin $) \cap L_{p}(G)$, и обозначим через $G_{z}$ множество $\left\{x \in \mathbb{R}^{n}: x+H_{0 z}^{t} \subset G\right\}$. Применяя $(22)$ к функции $u(\xi)=g(\xi+x)$, имеем при $x \in G_{z}$

$$
g(x)-\sum_{a \in M_{t}} P_{a}(-z) g(x+z+a)=\int_{F} \sum_{i=0}^{t}(-1)^{i}\left(\begin{array}{l}
t \\
i
\end{array}\right) g(x+\xi+i \eta) d \psi,
$$

откуда по неравенству Минковского

$$
\begin{aligned}
& \left\|g(x)-\sum_{a \in M_{t}} P_{a}(-z) g(x+z+a)\right\|_{L_{p}\left(G_{z}\right)} \\
& \leqslant \int_{F}\left\|\sum_{i=0}^{t}(-1)^{i}\left(\begin{array}{c}
t \\
i
\end{array}\right) g(x+\xi+i \eta)\right\|_{L_{p}\left(G_{z}\right)} d|\psi| \leqslant A_{2} \omega_{t}(g, \delta)_{L_{p}(G)}
\end{aligned}
$$


(так как $\left.H_{0 z}^{t} \subset B(0, \delta)\right)$. Поскольку $W_{\infty}^{0}(G$, fin $) \cap L_{p}(G)$ плотно в $L_{p}(G)$ при $p<\infty$, неравенство между крайними выражениями в (23) сохраняет силу и при условиях леммы. Его можно переписать в эквивалентном виде

$$
\left\|g(x)-P_{t}(g, x+z, x)\right\|_{L_{p}\left(G_{z}\right)} \leqslant A_{2} \omega_{t}(g, \delta)_{L_{p}(G)} .
$$

При $p=\infty$ здесь уже содержится требуемая оценка. Если $p<\infty$, то, делая в интеграле замену $z=y-x$ и используя (24), получаем

$$
\begin{aligned}
\widetilde{\omega}_{t}(g, \delta)_{p, G} & =\left(\iint_{(x, y) \in X_{t}(G, \delta)}\left|g(x)-P_{t}(g, y, x)\right|^{p} d x d y\right)^{1 / p} \\
& =\left(\iint_{\substack{H_{0 z}^{t} \subset B(0, \delta) \\
x \in G_{z}}}\left|g(x)-P_{t}(g, x+z, x)\right|^{p} d x d z\right)^{1 / p} \leqslant A_{3} \omega_{t}(g, \delta)_{L_{p}(G)} .
\end{aligned}
$$

Отметим, что метод доказательства леммы 5 близок к методу доказательства теоремы 3 из работы [39], в которой, однако, на множество $G$ наложено условие выпуклости.

Лемма 6 [3; лемма 5]. Допустим, что $\rho>0, \lambda \geqslant C_{1} \rho, F \subset \lambda \Omega$, причем либо $F$, либо $(\lambda \Omega) \backslash F$ является ограниченным множеством, $\Phi \subset L_{2}^{m}(\lambda \Omega)$ и выполняются условия:

1) для любих $g \in \Phi, x \in \lambda \Omega$ найдется такое $y \in \lambda \bar{\Omega}$, что $g(y)=0 u$ $|x-y| \leqslant \rho$

2) если функция $g_{0}$ бесконечно дифференцируема и ее производные порядка $\leqslant m$ ограничены на $\lambda \Omega$, причем $g_{0}(x)$ постоянна на $(\lambda \Omega) \backslash V$ (әде $V$ некоторое ограниченное множество) и равна 1 на $F$, mо $g g_{0} \in \Phi$ для любого $g \in \Phi$

3) существует такое $u \in \Phi$, ито

$$
\int_{\lambda \Omega} \sum_{|\alpha|_{1}=m} \frac{m !}{\alpha !}\left(D^{\alpha} u\right)^{2} d x=\inf _{g \in \Phi} \int_{\lambda \Omega} \sum_{|\alpha|_{1}=m} \frac{m !}{\alpha !}\left(D^{\alpha} g\right)^{2} d x .
$$

Тогда для любого $r \in \mathbb{R}$

$$
\|u\|_{W_{2}^{m}\left(\sum_{F \lambda}(r)\right)} \leqslant C_{2} C_{3}^{r / \rho}(1+\rho)^{m}\left\|D^{m} u\right\|_{L_{2}(\lambda \Omega)} .
$$

Здесь $C_{i}=C_{i}(\Omega, m)>0$, причем $C_{3}<1$.

Введем еще несколько обозначений. Допустим, что $\bar{h}>0$ (случай $\bar{h}=0$ тривиален) и задано число $\mu>0$. Пусть $\varepsilon_{0}-$ константа $C_{1}(\Omega)$ из леммы 2 , $\delta_{0}=\max \left\{1, C_{1}(\Omega, m), C_{1}(\Omega, 2 m), C_{4}(\Omega, m), C_{4}(\Omega, 2 m)\right\}$, где $C_{1}, C_{4}$ - константы из леммы $4, \lambda_{0}$ - константа $C_{1}(\Omega, m)$ из леммы $6, \lambda=\delta_{0} /(\mu \bar{h}), \tilde{f}(x)=f(x / \lambda)$, $Z=\left\{x \in \frac{1}{2 n} \mathbb{Z}^{n}: \operatorname{dist}(x, \lambda \Omega)<1\right\}, V_{x}=(\lambda \Omega) \cap B(x, 1), V_{x}^{\prime}=(\lambda \Omega) \cap B(x, 3)$, $x^{\prime}$ - некоторая точка $V_{x}$ для любого $x \in Z, \Pi_{x}(r)=\Pi_{x^{\prime} \lambda}(r)(x \in Z, r \in \mathbb{R})$, $r_{x y}=\inf \left\{r \in \mathbb{R}: x^{\prime} \in \Pi_{y}(r)\right\}(x, y \in Z), s_{y}=s\left(\tilde{f} \varphi_{y}, \lambda \Delta, m, \lambda \Omega\right)(y \in Z ;$ здесь сплайн является решением вариационной задачи). Кроме того, сейчас мы определим константу $h^{*}$. В [3] также накладьвалось условие вида $\bar{h}<h_{1}$, где 
$h_{1}=h_{1}(\Omega, m)>0$, которьм обеспечиваются существование и единственность интерполяционного сплайна при $f \in L_{p}^{k}(\Omega)$ (следовательно, при $\bar{h}<h_{1}$ выполняется (9)). В [3; замечание 3$]$ установлено сушествование таких констант $h_{2}=h_{2}(\Omega)>0, \varkappa^{*}=\varkappa^{*}(\Omega)>0$, что для любого $h \in\left(0, h_{2}\right)$ найдется множество $U \subset \Omega \mathrm{c} \bar{h}(U)=h, \underline{h}(U)>\varkappa^{*} h$. Положим

$$
h^{*}=\min \left\{\frac{1}{2}, \frac{\varepsilon_{0}}{4}, \frac{1}{9 \lambda_{0}}, h_{1}, h_{2}\right\} .
$$

ЛЕмма 7. Если $z \in Z, 0<\mu \leqslant 1$ и либо $\mu \leqslant \underline{h}(\bar{h})^{-1}$, либо $W_{p}^{k} \rightarrow W_{2}^{m}, m o$

$$
\sum_{y \in Z}\left\|s_{y}\right\|_{W_{2}^{m}\left(V_{z}^{\prime}\right)} \leqslant C_{1} \sum_{i=1}^{\infty} C_{2}^{i}\|\tilde{f}\|_{W_{p}^{k}\left(\Pi_{z}(i)\right)},
$$

әде $C_{i}=C_{i}(\Omega, m, k, p, \mu)$, причем $0<C_{2}<1$.

ДокаЗАТЕльСтво. Здесь $A_{i}=A_{i}(\Omega, m, k, p, \mu)>0$. По лемме 2 с $\varepsilon=4 / \lambda$ $\left(\varepsilon \leqslant \varepsilon_{0}\right.$ в силу того, что $\bar{h}<\varepsilon_{0} / 4$ и $\left.\mu \leqslant 1 \leqslant \delta_{0}\right)$ пересечение $\lambda \Omega$ с любым шаром радиуса 4 содержится в некотором связном подмножестве $\lambda \Omega$ диаметра $\leqslant$ $A_{1}$, поэтому $V_{y}^{\prime} \subset(\lambda \Omega) \cap B\left(y^{\prime}, 4\right) \subset \Pi_{y}\left(A_{1}\right)$ при любом $y \in Z$, а так как $y^{\prime} \in$ $\Pi_{z}\left(r_{y z}+1\right)$, то

$$
V_{y}^{\prime} \subset \Pi_{z}\left(r_{y z}+A_{2}\right) \quad \forall y \in Z .
$$

Если бы при некотором $y \in Z$ выполнялось условие

$$
V_{z}^{\prime} \cap \Pi_{V_{y} \lambda}\left(\frac{1}{2} r_{y z}-\frac{3}{2} A_{1}\right) \neq \varnothing,
$$

то, поскольку $V_{z}^{\prime} \subset \Pi_{z}\left(A_{1}\right)$ и $\Pi_{V_{y} \lambda}\left(\frac{1}{2} r_{y z}-\frac{3}{2} A_{1}\right) \subset \Pi_{y}\left(\frac{1}{2} r_{y z}-\frac{1}{2} A_{1}\right)$ (последнее в силу $\left.V_{y} \subset \Pi_{y}\left(A_{1}\right)\right)$, мы имели бы

$$
\Pi_{z}\left(A_{1}\right) \cap \Pi_{y}\left(\frac{1}{2} r_{y z}-\frac{1}{2} A_{1}\right) \neq \varnothing,
$$

откуда следует $y^{\prime} \in \Pi_{y}\left(\frac{1}{2} r_{y z}-\frac{1}{2} A_{1}\right) \subset \Pi_{z}\left(A_{1}+2\left(\frac{1}{2} r_{y z}-\frac{1}{2} A_{1}\right)\right)=\Pi_{z}\left(r_{y z}\right)$, что неверно. Итак,

$$
V_{z}^{\prime} \subset \Sigma_{V_{y} \lambda}\left(\frac{1}{2} r_{y z}-\frac{3}{2} A_{1}\right) \quad \forall y \in Z .
$$

Далее, заметим, что

$$
\sup _{x \in \Omega} \inf _{a \in \Delta \backslash B\left(y, \lambda^{-1}\right)}|x-a| \leqslant 9 \bar{h} \quad \forall y \in \mathbb{R}^{n} .
$$

Действительно, если $x \in \Omega \backslash B(y, 3 \bar{h})$, то $|x-a|<2 \bar{h}$ для некоторого $a \in \Delta$, и тогда $a \in \Delta \backslash B(y, \bar{h}) \subset \Delta \backslash B\left(y, \lambda^{-1}\right)$. Если же $x \in \Omega \cap B(y, 3 \bar{h})$, то ввиду $\operatorname{diam} \Omega \geqslant 2 \varepsilon_{0}>6 \bar{h}$ найдется точка $b \in \Omega \cap B(y, 4 \bar{h}) \backslash B(y, 3 \bar{h})$; для некоторого $a \in \Delta$ имеем $|a-b|<2 \bar{h}$, следовательно, $a \in \Delta \backslash B(y, \bar{h})$ и

$$
|x-a| \leqslant|x-y|+|y-b|+|b-a| \leqslant 3 \bar{h}+4 \bar{h}+2 \bar{h}=9 \bar{h} .
$$


Теперь, применяя лемму 6 с $\rho=9 \bar{h} \lambda\left(\lambda \geqslant \lambda_{0} \rho\right.$, так как $\left.\bar{h}<1 /\left(9 \lambda_{0}\right)\right), F=V_{y}$, $u=s_{y}$ и учитывая $(26),(27)$, имеем

$$
\left\|s_{y}\right\|_{W_{2}^{m}\left(V_{z}^{\prime}\right)} \leqslant A_{3} A_{4}^{r_{y z}}\left\|D^{m} s_{y}\right\|_{L_{2}(\lambda \Omega)}
$$

где $A_{4}<1$. Если $\mu \leqslant \underline{h}(\bar{h})^{-1}$, то $\lambda \underline{h} \geqslant 1$, так что по определению $s_{y}$ и по лемме 1

$$
\begin{aligned}
\left\|D^{m} s_{y}\right\|_{L_{2}(\lambda \Omega)} & \leqslant A_{5}\left\|D^{m} \sum_{a \in \lambda \Delta} \tilde{f}(a) \varphi_{y}(a) \varphi\left(\frac{x-a}{\lambda \underline{h}}\right)\right\|_{L_{2}(\lambda \Omega)} \\
& \leqslant A_{6}\|\tilde{f}\|_{L_{\infty}\left(V_{y}\right)} \leqslant A_{7}\|\tilde{f}\|_{W_{p}^{k}\left(V_{y}^{\prime}\right)}
\end{aligned}
$$

а в случае $W_{p}^{k} \rightarrow W_{2}^{m}$

$$
\begin{aligned}
\left\|D^{m} s_{y}\right\|_{L_{2}(\lambda \Omega)} & \leqslant A_{8}\left\|D^{m}\left(\tilde{f} \varphi_{y}\right)\right\|_{L_{2}(\lambda \Omega)} \\
& \leqslant A_{9}\|\tilde{f}\|_{W_{2}^{m}\left(V_{y}\right)} \leqslant A_{10}\|\tilde{f}\|_{W_{p}^{k}\left(V_{y}^{\prime}\right)} .
\end{aligned}
$$

Наконец, из (25) и (28)-(30) получаем

$$
\begin{aligned}
\sum_{y \in Z}\left\|s_{y}\right\|_{W_{2}^{m}\left(V_{z}^{\prime}\right)} & \leqslant \sum_{y \in Z} A_{11} A_{4}^{r_{y z}}\|\tilde{f}\|_{W_{p}^{k}\left(\Pi_{z}\left(r_{y z}+A_{2}\right)\right)} \\
& =A_{11} \sum_{i=1}^{\infty} \sum_{\substack{y \in Z \\
i-1 \leqslant r_{y z}<i}} A_{4}^{r_{y z}}\|\tilde{f}\|_{W_{p}^{k}\left(\Pi_{z}\left(r_{y z}+A_{2}\right)\right)} \\
& \leqslant A_{12} \sum_{i=1}^{\infty} i^{n} A_{4}^{i}\|\tilde{f}\|_{W_{p}^{k}\left(\Pi_{z}\left(i+A_{2}\right)\right)} \\
& \leqslant A_{13} \sum_{i=1}^{\infty} A_{14}^{i}\|\tilde{f}\|_{W_{p}^{k}\left(\Pi_{z}(i)\right)}
\end{aligned}
$$

где $A_{14}<1$.

Лемма 8. Пусть $0<\mu \leqslant 1,0<\gamma<1, U \subset \lambda \Omega, r(x)=\operatorname{dist}(x, U)$, $Z^{\prime}=\left\{z \in Z: V_{z} \cap U \neq \varnothing\right\}, g \in L_{p}(\lambda \Omega), t \in \mathbb{Z}_{+} u$

$$
N_{z}=\sum_{i=1}^{\infty} \gamma^{i} \widetilde{\omega}_{t}(g, \delta)_{p, \Pi_{z}(i)}
$$

Тогда

$$
\left(\sum_{z \in Z^{\prime}} N_{z}^{q}\right)^{1 / q} \leqslant C \lambda^{\left(\frac{n}{q}-\frac{n}{p}\right)+}\left\|\left(g(x)-P_{t}(g, y, x)\right) \gamma^{\frac{r(x)}{2 p}}\right\|_{L_{p}\left(X_{t}(\lambda \Omega, \delta)\right)},
$$

где $C=C(\Omega, \gamma)$ и в случае $q=\infty$ в левой части стоит $\sup _{z \in Z^{\prime}} N_{z}$. 
ДоказАТЕЛЬСтво. Ниже $A_{i}=A_{i}(\Omega, \gamma)>0$. Используя неравенство

$$
\left(\sum_{i=0}^{\infty} a_{i} \gamma^{i}\right)^{p} \leqslant(1-\gamma)^{1-p} \sum_{i=0}^{\infty} a_{i}^{p} \gamma^{i}
$$

где $a_{i} \geqslant 0$, и учитывая, что в случае $p>q$ множество $\Omega$ ограничено, $\lambda \geqslant 1$, запишем

$$
\begin{aligned}
& \left(\sum_{z \in Z^{\prime}} N_{z}^{q}\right)^{1 / q} \lambda^{-\left(\frac{n}{q}-\frac{n}{p}\right)_{+}} \\
& \quad \leqslant A_{1}\left(\sum_{z \in Z^{\prime}} N_{z}^{p}\right)^{1 / p} \leqslant A_{2}\left(\sum_{z \in Z^{\prime}} \sum_{i=1}^{\infty} \gamma^{i} \widetilde{\omega}_{t}(g, \delta)_{p, \Pi_{z}(i)}^{p}\right)^{1 / p} \\
& \quad=A_{2}\left(\iint_{(x, y) \in X_{t}(\lambda \Omega, \delta)}\left|g(x)-P_{t}(g, y, x)\right|^{p} \psi(x, y) d x d y\right)^{1 / p}
\end{aligned}
$$

где $\psi(x, y)=\sum \gamma^{i}$, сумма распространяется на пары $(z, i) \in Z \times \mathbb{N}$ такие, что $V_{z} \cap U \neq \varnothing, H_{x y}^{t} \subset \Pi_{z}(i)$. Так как при этих условиях $i>|x-z|-1>r(x)-2$ и количество пар с данным $i$ не превосходит $A_{3} i^{n}$, имеем

$$
\psi(x, y) \leqslant \sum_{i>r(x)-2} A_{3} i^{n} \gamma^{i} \leqslant A_{4} \gamma^{r(x) / 2}
$$

что и дает (31). Здесь мы формально предполагали, что $p, q<\infty$, но это ограничение, очевидно, несущественно.

ДОКАЗАТЕЛЬСТВо ТЕОРЕМЫ 1 . Положим $\mu=\min \{1, \varkappa\}$ в случае 1$)$ и $\mu=1$ в случае 2) (см. формулировку теоремы). Далее $A_{i}=A_{i}(\Omega, m, p, q, \mu)>0$. Обозначим сумму $\sum_{y \in Z} s_{y}$ через $\tilde{s}$. Если $Z$ бесконечно, то ряд безусловно сходится в $W_{2}^{m}(\lambda \Omega$, fin $)$, что следует из леммы 7 и оценки

$$
\|\tilde{f}\|_{W_{p}^{k}\left(\Pi_{a}(\rho)\right)} \leqslant A^{\prime} \rho^{A_{1}},
$$

где $\rho \geqslant 1, a \in \lambda \Omega, A^{\prime}=A^{\prime}(\Omega, k, f, \lambda, a)$, которая вытекает из леммы 4. Выполнение условий 1$), 2)$ из определения сплайна для функции $s(x)=\tilde{s}(\lambda x)$ очевидно; проверим условие 3$)$. Пусть $a \in \lambda \Omega, r \geqslant 1, z \in Z, V_{z} \cap \Pi_{a}(r) \neq \varnothing$. По лемме 2 с $\varepsilon=4 / \lambda$ $\left(\varepsilon<\varepsilon_{0}\right.$, так как $\left.\bar{h}<\varepsilon_{0} / 4, \mu \leqslant 1 \leqslant \delta_{0}\right)$

$$
V_{z}^{\prime} \subset \Pi_{z}\left(A_{2}\right),
$$

так что $z^{\prime} \in \Pi_{a}\left(r+A_{3}\right)$, поэтому $\Pi_{z}(i) \subset \Pi_{a}\left(i+r+A_{3}\right) \forall i \in \mathbb{N}$. Отсюда, из леммы 7 и неравенства (32) следует, что $\|\tilde{s}\|_{W_{2}^{m}\left(V_{z}\right)} \leqslant A_{4} A^{\prime} r^{A_{1}}$. Если просуммировать эти оценки по всем $z \in Z$ таким, что $V_{z}$ пересекает $\Pi_{a}(r)$ (количество этих $z$ не превосходит $\left.A_{5} r^{n}\right)$, получится $\|\tilde{s}\|_{W_{2}^{m}\left(\Pi_{a}(r)\right)}=O\left(r^{A_{6}}\right)$ при $r \rightarrow \infty$. Итак,

$$
s(f, \Delta, m, \Omega)\left(\frac{x}{\lambda}\right)=\sum_{y \in Z} s\left(\tilde{f} \varphi_{y}, \lambda \Delta, m, \lambda \Omega\right)(x),
$$

где при бесконечном $Z$ ряд безусловно сходится в $W_{2}^{m}(\lambda \Omega, \mathrm{fin})$. 
Если $f$ - полином степени $<m$, то, очевидно, $s(f, \Delta, m, \Omega)=f$. В случае $\Omega=$ $\mathbb{R}^{n}$ это верно для полиномов степени $<2 m$, так как если $f \in \mathscr{P}_{2 m-1}, g \in W_{2}^{m}\left(\mathbb{R}^{n}\right)$, $g$ имеет ограниченный носитель, то

$$
\int_{\mathbb{R}^{n}} \sum_{|\alpha|_{1}=m} \frac{m !}{\alpha !} D^{\alpha} f D^{\alpha} g d x=(-1)^{m} \int_{\mathbb{R}^{n}} \mathscr{L}^{m} f \cdot g d x=0 .
$$

Пусть $t=m$ при $\Omega \neq \mathbb{R}^{n}$ и $t=2 m$ при $\Omega=\mathbb{R}^{n}, z \in Z, P$ - полином степени $<t$, соответствующий функции $g=\tilde{f}$ согласно лемме 4 при $a=z^{\prime}\left(\lambda \geqslant \delta_{0}\right)$. На основании вышесказанного

$$
\tilde{s}-P=\sum_{y \in Z} s_{y}-\sum_{y \in Z} s\left(P \varphi_{y}, \lambda \Delta, m, \lambda \Omega\right)=\sum_{y \in Z} s\left((\tilde{f}-P) \varphi_{y}, \lambda \Delta, m, \lambda \Omega\right) .
$$

Отсюда по леммам 1 и 7 (с заменой $\tilde{f}$ на $\tilde{f}-P$ ) получаем

$$
\begin{aligned}
\left\|D^{l}(\tilde{s}-P)\right\|_{L_{q}\left(V_{z}\right)} & \leqslant A_{7}\|\tilde{s}-P\|_{W_{2}^{m}\left(V_{z}^{\prime}\right)} \\
& \leqslant A_{8} \sum_{i=1}^{\infty} A_{9}^{i}\|\tilde{f}-P\|_{W_{p}^{k}\left(\Pi_{z}(i)\right)} \\
& \leqslant A_{10} \sum_{i=1}^{\infty} A_{11}^{i} \widetilde{\omega}_{t-k}\left(D^{k} f, \delta_{0}\right)_{p, \Pi_{z}(i)}
\end{aligned}
$$

где $A_{9}, A_{11}<1$. Кроме того, в силу леммы 1 и $(33)$

$$
\left\|D^{l}(\tilde{f}-P)\right\|_{L_{q}\left(V_{z}\right)} \leqslant A_{12}\|\tilde{f}-P\|_{W_{p}^{k}\left(V_{z}^{\prime}\right)} \leqslant A_{13} \widetilde{\omega}_{t-k}\left(D^{k} \tilde{f}, \delta_{0}\right)_{p, \Pi_{z}\left(A_{14}\right)},
$$

так что

$$
\left\|D^{l}(\tilde{f}-\tilde{s})\right\|_{L_{q}\left(V_{z}\right)} \leqslant A_{15} \sum_{i=1}^{\infty} A_{11}^{i} \widetilde{\omega}_{t-k}\left(D^{k} \tilde{f}, \delta_{0}\right)_{p, \Pi_{z}(i)} .
$$

Используя это неравенство, а также леммы 5 и 8 (здесь $U=\lambda \Omega$ ), находим

$$
\begin{aligned}
\left\|D^{l}(\tilde{f}-\tilde{s})\right\|_{L_{q}(\lambda \Omega)} & \leqslant A_{16}(\bar{h})^{-\left(\frac{n}{q}-\frac{n}{p}\right)_{+}} \widetilde{\omega}_{t-k}\left(D^{k} \tilde{f}, \delta_{0}\right)_{p, \lambda \Omega} \\
& \leqslant A_{17}(\bar{h})^{-\left(\frac{n}{q}-\frac{n}{p}\right)_{+}} \omega_{t-k}\left(D^{k} \tilde{f}, \delta_{0}\right)_{L_{p}(\lambda \Omega)} .
\end{aligned}
$$

Остается применить преобразование подобия и перейти к функциям на $\Omega$.

ЗАмечание 1 . Пусть выполнены условия теоремы $1, k=m, p<\infty, \Omega \neq \mathbb{R}^{n}$, $\Omega^{\prime}$ - открытое подмножество $\Omega$. Если в конце доказательства теоремы 1 применить лемму 8 для $U=\lambda \Omega^{\prime}$, получим неравенство

$$
\left\|D^{l}(f-s)\right\|_{L_{q}\left(\Omega^{\prime}\right)} \leqslant C_{1} \bar{h}^{\theta} \max _{|\alpha|_{1}=k}\left\|D^{\alpha} f(x) C_{2}^{\rho(x)}\right\|_{L_{p}(\Omega)}
$$

где $\rho(x)=\operatorname{dist}\left(x, \Omega^{\prime}\right) / \bar{h}, C_{i}=C_{i}(\Omega, m, p, q, \varkappa)$ в случае 1$)$ теоремы 1 и $C_{i}=$ $C_{i}(\Omega, m, p, q)$ в случае 2$), 0<C_{2}<1$. 
Замечание 2 . Пусть $n=1, \Omega$ ограничено, $\partial \Omega \subset \Delta$. Тогда теорема 1 и замечание 1 в случае 1 ) останутся в силе, если заменить в них условие $W_{2}^{m} \rightarrow W_{q}^{l}$ на более слабое условие $l<2 m$. Действительно, вложение $W_{2}^{m} \rightarrow W_{q}^{l}$ использовалось в доказательстве теоремы 1 только для того, чтобы оценить $\left\|D^{l}(\tilde{s}-P)\right\|_{L_{q}\left(V_{z}\right)}$ через $\|\tilde{s}-P\|_{W_{2}^{m}\left(V_{z}^{\prime}\right)}$ см. (34). А поскольку сплайн $s(x)$ является кусочно полиномиальной функцией степени $<2 m$, точки "склейки" которой принадлежат $\Delta, s \in W_{\infty}^{2 m-2}(\Omega)($ см. [23]) и $\lambda \underline{h} \geqslant 1$, то первое из неравенств (34) выполняется и при $l<2 m$.

Теперь допустим, что $\Omega$ ограничено, $k<m$ и $W_{2}^{m} \rightarrow W_{p}^{k}$. Положим $F_{i}=$ $\left(\left(2^{-i} \mathbb{Z}^{n}\right) \backslash\left(2^{-i+1} \mathbb{Z}^{n}\right)\right) \cap \Omega$ и представим множество $\bigcup_{i=1}^{\infty} F_{i}$ в виде последовательности $\left\{a_{1}, a_{2}, \ldots\right\}$ так, чтобы сначала шли элементы $F_{1}$, затем- элементы $F_{2}$ и т. д. Пусть $\Delta_{i}=\left\{a_{1}, \ldots, a_{i}\right\}, u_{i}$ - некоторый элемент $W_{2}^{m}(\Omega)$, минимизирующий функционал $\int_{\Omega} \sum_{|\alpha|_{1}=m} \frac{m !}{\alpha !}\left(D^{\alpha} g\right)^{2} d x$ при условиях: $g\left(a_{i}\right)=1, g\left(a_{j}\right)=0, j<i$. Тогда функции $u_{i}, i \in \mathbb{N}$, образуют базис в $W_{p}^{k}(\Omega)$, причем для достаточно больших $N$

$$
\sum_{i=1}^{N} \lambda_{i} u_{i}=s\left(f, \Delta_{N}, m, \Omega\right)
$$

где $\sum_{i=1}^{\infty} \lambda_{i} u_{i}-$ разложение функции $f \in W_{p}^{k}(\Omega)$ по этому базису [3]. Поскольку величины $\bar{h}\left(\Delta_{N}\right), \underline{h}\left(\Delta_{N}\right)$ заключены между $C_{1} N^{-1 / n}$ и $C_{2} N^{-1 / n}$, где $C_{i}=$ $C_{i}(\Omega)>0$, из теоремы 1 вытекает

СлЕДСТвИЕ 1. При достаточно больиих $N$

$$
\sum_{l=0}^{k}\left\|D^{l}\left(f-\sum_{i=1}^{N} \lambda_{i} u_{i}\right)\right\|_{L_{p}(\Omega)} N^{(k-l) / n} \leqslant C \omega_{m-k}\left(D^{k} f, N^{-1 / n}\right)_{L_{p}(\Omega)},
$$

әде $C=C(\Omega, m, p)$.

(Здесь использована оценка $\omega_{t}(g, 2 \delta)_{L_{p}(\Omega)} \leqslant 2^{t} \omega_{t}(g, \delta)_{L_{p}(\Omega)}$, см. [38].)

\section{§2. Оценки на $\Omega_{(\varepsilon)}$}

В предыдушем параграфе оценки погрештости сплайн-интерполяции на всей области $\Omega$ были получены для $\Omega=\mathbb{R}^{n}$ при $k \leqslant 2 m$, а в случае, когда $\Omega$ имеет границу, - лишь при $k \leqslant m$. Дело здесь не только в том, что в случае $\Omega=\mathbb{R}^{n}$ оператор $f \rightarrow s(f, \Delta, m, \Omega)$ сохраняет полиномы более высокой степени, чем при $\Omega \neq \mathbb{R}^{n}$. Есть другая причина: аппроксимативные свойства сплайнов $s(f, \Delta, m, \Omega)$ для достаточно гладких интерполируемых функций $f$ вблизи границы области $\Omega$ оказываются хуже, чем на расстоянии от границы. ${ }^{6}$ Это связано с тем, что сплайн $s(x)$ удовлетворяет некоторым естественным краевым условиям, которые, например, в случае $n=1, \Omega=(0,1)$ имеют вид $s^{(j)}(0)=s^{(j)}(1)=0$ при $j=\overline{m, 2 m-2}$. В этом параграфе мы получим оценку величины $\left\|D^{l}(f-s)\right\|_{L_{q}\left(\Omega_{(\varepsilon)}\right)}, \varepsilon>0$, иного вида, чем (10), при $k \leqslant 2 m$. Для упрощения рассуждений будем считать, что область $\Omega$ ограничена. Добавим, что на расстоянии от границы и гладкость сплайна

\footnotetext{
${ }^{6}$ В одномерном случае это обнаружил Т. Лукас [19] при $m=2$.
} 
вьше, чем $W_{2}^{m}$, если $\underline{h}(\Delta)>0$ : мы покажем, что в этом случае $s \in W_{q}^{l}\left(\Omega_{(\varepsilon)}\right)$ при $l-n / q<2 m-n, \varepsilon>0$. Кроме того, будет получена точная по порядку оценка сходимости сплайнов на $\Omega_{(\varepsilon)}$, т.е. мы найдем наименьшую (в порядковом смысле) функцию $\chi(h)$, удовлетворяющую условию: для любого $f \in W_{p}^{k}(\Omega)$ и любой последовательности множеств $\Delta_{i} \subset \bar{\Omega}, i \in \mathbb{N}$, с $\bar{h}\left(\Delta_{i}\right) \rightarrow 0$ имеем $\left\|D^{l}\left(f-s\left(f, \Delta_{i}, m, \Omega\right)\right)\right\|_{L_{q}\left(\Omega_{(\varepsilon)}\right)}=O\left(\chi\left(\bar{h}\left(\Delta_{i}\right)\right)\right)$ при $i \rightarrow \infty$. Сейчас заметим, что если для некоторой функции $\chi(h)$ это условие выполняется, то множество линейных операторов $\left\{f \rightarrow \chi\left(\bar{h}\left(\Delta_{i}\right)\right)^{-1} D^{\alpha}\left(f-s\left(f, \Delta_{i}, m, \Omega\right)\right): i \in \mathbb{N},|\alpha|=l\right\}$ из $W_{p}^{k}(\Omega)$ в $L_{q}\left(\Omega_{(\varepsilon)}\right)$ поточечно ограничено, и если эти операторы непрерывны (что гарантируется при соответствуюших условиях теоремой 2), то по теореме Банаха-Штейнгауза существуют такие $h_{0}>0$ и $C$, не зависящие от $f, \Delta$, что при $\bar{h}(\Delta)<h_{0}$

$$
\left\|D^{l}(f-s(f, \Delta, m, \Omega))\right\|_{L_{q}\left(\Omega_{(\varepsilon)}\right)} \leqslant C \chi(\bar{h}(\Delta))\|f\|_{W_{p}^{k}(\Omega)} .
$$

Данньй вопрос, таким образом, сводится к исследованию величины

$$
\sup \left\{\left\|D^{l}(f-s(f, \Delta, m, \Omega))\right\|_{L_{q}\left(\Omega_{(\varepsilon)}\right)}:\|f\|_{W_{p}^{k}(\Omega)} \leqslant 1, \bar{h}(\Delta)=h\right\}
$$

которую мы в дальнейшем будем обозначать через $\chi(h)$. Аналогично, если рассматривается сходимость сплайнов при дополнительном условии квазиравномерности последовательности $\left\{\Delta_{i}\right\}: \bar{h}\left(\Delta_{i}\right)=O\left(\underline{h}\left(\Delta_{i}\right)\right)$ при $i \rightarrow \infty$, то этой ситуации соответствует величина

$\chi_{\varkappa}(h)=\sup \left\{\left\|D^{l}(f-s(f, \Delta, m, \Omega))\right\|_{L_{q}\left(\Omega_{(\varepsilon)}\right)}:\|f\|_{W_{p}^{k}(\Omega)} \leqslant 1, \bar{h}(\Delta)=h, \underline{h}(\Delta)>\varkappa h\right\}$,

где $\varkappa>0$.

ТЕОРема 2. Предположим, что $k \leqslant 2 m, \varepsilon>0$ и выполнено одно из двух условий:

1) $l-n / q<2 m-n u(4)$;

2) $W_{p}^{k} \rightarrow W_{2}^{m} \rightarrow W_{q}^{l}$.

Тогда

$$
\left\|D^{l}(f-s)\right\|_{L_{q}\left(\Omega_{(\varepsilon)}\right)} \leqslant C_{1}\left(\bar{h}^{\theta} \omega_{2 m-k}\left(D^{k} f, \bar{h}\right)_{L_{p}(\Omega)}+C_{2}^{1 / \bar{h}} \omega_{m}(f, \bar{h})_{L_{1}(\Omega)}\right)
$$

где $C_{i}=C_{i}(\Omega, m, p, q, \varepsilon, \varkappa)$ в случае 1$), C_{i}=C_{i}(\Omega, m, p, q, \varepsilon)$ в случае 2) и $0<C_{2}<1$.

Сначала докажем две леммы.

Лемма 9. Если $\lambda>0, B(a, 2) \subset \lambda \Omega, \Delta^{\prime}=(\lambda \Delta) \cap B(a, 2),\left(x, y \in \Delta^{\prime}\right) \Rightarrow$ $(|x-y| \geqslant 1), l-n / q<2 m-n u \tilde{s}(x)=s(x / \lambda)$, mo $\tilde{s} \in W_{q}^{l}(B(a, 1)) u$

$$
\|\tilde{s}\|_{W_{q}^{l}(B(a, 1))} \leqslant C\|\tilde{s}\|_{L_{1}(B(a, 2))},
$$

əде $C=C(m, q)$ 
ДоКАЗАТЕЛЬСТво. В силу условия 1) из определения сплайна сушествуют такие $\xi_{y} \in \mathbb{R}\left(y \in \Delta^{\prime}\right)$, что для любой функции $g \in \mathscr{D}(B(a, 2))$

$$
\int_{B(a, 2)} \tilde{s} \cdot \mathscr{L}^{m} g d x=(-1)^{m} \int_{B(a, 2)} \sum_{|\alpha|_{1}=m} \frac{m !}{\alpha !} D^{\alpha} \tilde{s} D^{\alpha} g d x=\sum_{y \in \Delta^{\prime}} \xi_{y} g(y) .
$$

Функция $K(x-y)$ совпадает (с точностью до постоянного множителя) с фундаментальным решением уравнения $\mathscr{L}^{m} u(x)=0$ (см. [41]), поэтому при соответствующих $\xi_{y}^{\prime}$

$$
\int_{B(a, 2)}\left(\tilde{s}(x)-\sum_{y \in \Delta^{\prime}} \xi_{y}^{\prime} K(x-y)\right) \mathscr{L}^{m} g(x) d x=0 \quad \forall g \in \mathscr{D}(B(a, 2)) .
$$

Отсюда следует (см. [42]), что

$$
\tilde{s}(x)=\sum_{y \in \Delta^{\prime}} \xi_{y}^{\prime} K(x-y)+v(x)
$$

где $v$ - бесконечно дифференцируемая функция, полигармоническая порядка $m$ на $B(a, 2)$. Поскольку функция $K(x)$ - полигармоническая порядка $m$ на $\mathbb{R}^{n} \backslash\{0\}$, $K \in W_{q}^{l}(B(0,1))$ (при $l-n / q<2 m-n$ ) и имеет место представление (36), нам достаточно установить неравенство

$$
\|\xi K+g\|_{W_{q}^{l}(B(0,1))} \leqslant A_{1}\|\xi K+g\|_{L_{1}(B(0,2))},
$$

где $\xi \in \mathbb{R}, \mathscr{L}^{m} g(x)=0$ на $B(0,2)$ и $A_{1}=A_{1}(m, q)$. Заметим, что если $\mathscr{L}^{m} g(x)=0$ на $B(0,1)$, то для любого мультииндекса $\alpha$

$$
\left|D^{\alpha} g(0)\right| \leqslant A_{2}\|g\|_{L_{1}(B(0,1))},
$$

где $A_{2}=A_{2}(m, \alpha)$ (это следует из сушествования функции $\psi \in \mathscr{D}\left(B\left(0, \frac{1}{2}\right)\right)$, зависящей только от $m, n$, такой, что $g=g * \psi$ на $B\left(0, \frac{1}{2}\right)$, см. [42]). Допустим, что неравенство (37) не вьполняется. Тогда существуют такие $\xi_{i} \in \mathbb{R}$ и $g_{i}(x)(i \in \mathbb{N})$, что $\mathscr{L}^{m} g_{i}(x)=0$ на $B(0,2)$,

$$
\lim _{i \rightarrow \infty}\left\|\xi_{i} K+g_{i}\right\|_{W_{q}^{l}(B(0,1))}=\infty, \quad \lim _{i \rightarrow \infty}\left\|\xi_{i} K+g_{i}\right\|_{L_{1}(B(0,2))}=0 .
$$

В случае $\underline{\lim }_{i \rightarrow \infty}\left|\xi_{i}\right|=0$ получаем противоречие с (38), а в противном случае $\lim _{i \rightarrow \infty}\left\|\bar{K}_{i} \xi_{i}^{-1}\right\|_{L_{1}(B(0,2))}=0$, откуда ввиду (38) следует бесконечная дифференцируемость $K$ на $B(0,1)$, что неверно.

Лемма 10. Допустим, что выполняются условия теоремы 2 и $W_{2}^{m} \rightarrow W_{q}^{l}$. Тогда

$$
\begin{aligned}
\left\|D^{l}(f-s)\right\|_{L_{q}\left(\Omega_{(\varepsilon)}\right)} \leqslant & C_{1} \bar{h}^{\theta} \omega_{2 m-k}\left(D^{k} f, \bar{h}\right)_{L_{p}(\Omega)} \\
& +C_{1} \varepsilon^{-C_{2}} C_{3}^{\varepsilon / \bar{h}}\left(\omega_{2 m-k}\left(D^{k} f, \bar{h}\right)_{L_{p}(\Omega)}+\omega_{m}(f, \bar{h})_{L_{1}(\Omega)}\right),
\end{aligned}
$$

где $C_{i}=C_{i}(\Omega, m, p, q, \varkappa)$ в случае 1$)$ теоремы 2 и $C_{i}=C_{i}(\Omega, m, p, q)$ в случае 2$)$, $C_{i}>0, C_{3}<1$. 
ДоКАЗАТЕЛЬСТво. Здесь $A_{i}=A_{i}(\Omega, m, p, q, \varkappa)$ в случае 1$)$ и $A_{i}=A_{i}(\Omega, m, p, q)$ в случае 2$) ; A_{i}>0$. Мы будем использовать обозначения $\varepsilon_{0}, \delta_{0}, \lambda, \tilde{f}, Z, V_{x}, V_{x}^{\prime}, x^{\prime}$, $\Pi_{x}(r)$, введенные в $\S 1$. Пусть $\mu=\min \left\{\frac{1}{2}, \varkappa\right\}$ в случае 1) и $\mu=\frac{1}{2}$ в случае 2$), z \in Z$, $V_{z} \cap\left(\lambda \Omega_{(\varepsilon)}\right) \neq \varnothing, P$ - полином, соответствующий согласно лемме 4 функции $g=\tilde{f}$ при $a=z^{\prime}, t=2 m\left(\lambda \geqslant \delta_{0}\right)$, и $\tilde{s}(x)=s(x / \lambda)$. Очевидно, $\tilde{f}-\tilde{s}=R_{1}+R_{2}+R_{3}$, где $R_{1}=\tilde{f}-P, R_{2}=s(P-\tilde{f}, \lambda \Delta, m, \lambda \Omega), R_{3}=P-s(P, \lambda \Delta, m, \lambda \Omega)$. Заметим, что $V_{z}^{\prime} \subset \Pi_{z}\left(A_{1}\right)$ (лемма $\left.2,4 / \lambda<\varepsilon_{0}\right)$. Отсюда и из лемм 1,7 следуют неравенства

$$
\begin{aligned}
& \left\|R_{1}\right\|_{W_{q}^{l}\left(V_{z}\right)} \leqslant A_{2}\left\|R_{1}\right\|_{W_{p}^{k}\left(V_{z}^{\prime}\right)} \leqslant A_{3} \widetilde{\omega}_{2 m-k}\left(D^{k} \tilde{f}, \delta_{0}\right)_{p, \Pi_{z}\left(A_{4}\right)}, \\
& \left\|R_{2}\right\|_{W_{q}^{l}\left(V_{z}\right)} \leqslant A_{5}\left\|R_{2}\right\|_{W_{2}^{m}\left(V_{z}^{\prime}\right)} \leqslant A_{6} \sum_{i=1}^{\infty} A_{7}^{i} \widetilde{\omega}_{2 m-k}\left(D^{k} \tilde{f}, \delta_{0}\right)_{p, \Pi_{z}(i)},
\end{aligned}
$$

где $A_{7}<1$. Положим $F=\lambda \Omega^{(\varepsilon / 2)}$ и $R_{4}=s\left(R_{3},(\lambda \Delta) \cup F, m, \lambda \Omega\right)$. Применяя лемму 6 при указанном $F, \rho=\lambda \bar{h}$ и учитывая, что $R_{3}(x)=0$ на $\lambda \Delta, \operatorname{dist}\left(F, V_{z}^{\prime}\right) \geqslant$ $\lambda \varepsilon / 2-4 \geqslant \varepsilon / \bar{h}-4$, получаем

$$
\begin{aligned}
\left\|R_{4}\right\|_{W_{q}^{l}\left(V_{z}\right)} & \leqslant A_{8}\left\|R_{4}\right\|_{W_{2}^{m}\left(V_{z}^{\prime}\right)} \leqslant A_{9} A_{10}^{\varepsilon / \bar{h}}\left\|D^{m} R_{3}\right\|_{L_{2}(\lambda \Omega)} \\
& \leqslant A_{11} A_{10}^{\varepsilon / \bar{h}}\left\|D^{m} P\right\|_{L_{2}(\lambda \Omega)},
\end{aligned}
$$

где $A_{10}<1$. Поскольку $\left\|D^{m} \cdot\right\|_{L_{1}(B(0,1))}$ и $\widetilde{\omega}_{m}(\cdot, 2)_{1, B(0,1)}$ - две эквивалентные полунормы на $\mathscr{P}_{2 m-1}, \Pi_{z}\left(A_{12}\right)$ содержит некоторьй шар $B$ радиуса 1 (лемма 2 , $\left.1 / \lambda<\varepsilon_{0}\right)$, имеем

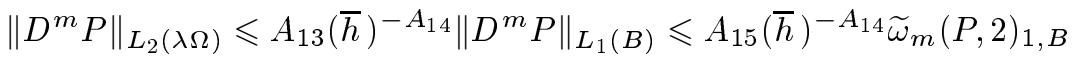

$$
\begin{aligned}
& \leqslant A_{16}(\bar{h})^{-A_{14}}\left(\widetilde{\omega}_{m}(\tilde{f}, 2)_{1, B}+\|\tilde{f}-P\|_{L_{1}(B)}\right) \\
& \leqslant A_{17}(\bar{h})^{-A_{14}}\left(\widetilde{\omega}_{m}(\tilde{f}, 2)_{1, \Pi_{z}\left(A_{12}\right)}+\widetilde{\omega}_{2 m-k}\left(D^{k} \tilde{f}, \delta_{0}\right)_{p, \Pi_{z}\left(A_{18}\right)}\right) \text {. }
\end{aligned}
$$

Поскольку $P \in \mathscr{P}_{2 m-1}$, то для любой функции $g \in W_{2}^{m}(\lambda \Omega)$, равной нулю на $F$,

поэтому

$$
\int_{\lambda \Omega} \sum_{|\alpha|_{1}=m} \frac{m !}{\alpha !} D^{\alpha} P D^{\alpha} g d x=(-1)^{m} \int_{\lambda \Omega} \mathscr{L}^{m} P \cdot g d x=0
$$

На основании (39)-(43)

$$
R_{3}=R_{4}
$$

$$
\begin{aligned}
\|\tilde{f}-\tilde{s}\|_{W_{q}^{l}\left(V_{z}\right)} & =\left\|R_{1}+R_{2}+R_{3}\right\|_{W_{q}^{l}\left(V_{z}\right)} \\
\leqslant & A_{19}\left(\sum_{i=1}^{\infty} A_{7}^{i} \widetilde{\omega}_{2 m-k}\left(D^{k} \tilde{f}, \delta_{0}\right)_{p, \Pi_{z}(i)}\right. \\
& \left.+(\bar{h})^{-A_{14}} A_{10}^{\varepsilon / \bar{h}}\left(\widetilde{\omega}_{2 m-k}\left(D^{k} \tilde{f}, \delta_{0}\right)_{p, \Pi_{z}\left(A_{18}\right)}+\widetilde{\omega}_{m}(\tilde{f}, 2)_{1, \Pi_{z}\left(A_{12}\right)}\right)\right) .
\end{aligned}
$$

Отсюда, используя лемму 8 (здесь $\left.U=\lambda \Omega_{(\varepsilon)}\right)$ и неравенство Минковского в $l_{q}$, получаем

$$
\begin{aligned}
\left\|D^{l}(\tilde{f}-\tilde{s})\right\|_{L_{q}\left(\lambda \Omega_{(\varepsilon)}\right) \leqslant} & A_{20}\left((\bar{h})^{-\left(\frac{n}{q}-\frac{n}{p}\right)_{+}} \widetilde{\omega}_{2 m-k}\left(D^{k} \tilde{f}, \delta_{0}\right)_{p, \lambda \Omega}\right. \\
& \left.+(\bar{h})^{-A_{21}} A_{10}^{\varepsilon / \bar{h}}\left(\widetilde{\omega}_{2 m-k}\left(D^{k} \tilde{f}, \delta_{0}\right)_{p, \lambda \Omega}+\widetilde{\omega}_{m}(\tilde{f}, 2)_{1, \lambda \Omega}\right)\right) .
\end{aligned}
$$

Доказательство завершается применением леммы 5 и преобразования подобия. 
Замечание 3 . Пусть $n=1$ и $\partial \Omega \subset \Delta$. Тогда лемма 10 справедлива и без условия $W_{2}^{m} \rightarrow W_{q}^{l}$ (т.е. справедлива при условиях теоремы 2 ). Это условие было нужно только для того, чтобы оценить в $(40),(41)$ величину $\left\|R_{i}\right\|_{W_{q}^{l}\left(V_{z}\right)}$ через $\left\|R_{i}\right\|_{W_{2}^{m}\left(V_{z}^{\prime}\right)}, i=2,4$, а так как в случае 1$)$ теоремы 2 функции $R_{2}$ и $R_{4}=R_{3}$ кусочно полиномиальные (см. замечание 2 ) и $\lambda \underline{h} \geqslant 1$, первые неравенства в (40), (41) вьполняются и в данной ситуации.

ДОКАЗАТЕЛЬСТВо ТЕОРЕМЫ 2. Для того чтобы получить теорему 2 , нужно внести небольшие изменения в доказательство леммы 10. Поскольку случай 2) теоремы 2 содержится в лемме 10 , рассмотрим только случай 1$)$. Положим на этот раз $\mu=\min \left\{\frac{1}{2}, \frac{\varepsilon}{6}, \varkappa\right\}$ и будем считать, что $A_{i}=A_{i}(\Omega, m, p, q, \varepsilon, \varkappa)$, т.е. допустим зависимость констант $A_{i}$ от $\varepsilon$. Дальше все идет так же, как в доказательстве леммы 10 , только для получения первых неравенств в (40) и (41) надо использовать лемму 9 с учетом того, что $B(z, 2) \subset \lambda \Omega_{(\varepsilon / 2)}\left(\right.$ так как $\left.V_{z} \cap\left(\lambda \Omega_{(\varepsilon)}\right) \neq \varnothing, \lambda \varepsilon / 2>3\right)$ и $\lambda \underline{h} \geqslant 1$.

Положим

$$
\begin{gathered}
\theta^{\prime}=\theta^{\prime}(k, p)=\min \{\theta(k, p), 2 m-l\}, \\
T=\left\{\left(2 m+i, \frac{n}{i}, \infty\right): i \in \mathbb{N}, i<n\right\} .
\end{gathered}
$$

Обозначения $\varepsilon_{0}, \varkappa^{*}, h_{2}$ введены в $\S 1$.

TeOpema 3. Пусть $l-n / q<2 m-n, 0<\varepsilon<\varepsilon_{0} / 2,0<h<h^{*}, 0<\varkappa<\varkappa^{*} u$ $(k, p, q) \notin T$. Тогда

$$
C_{1} h^{\theta^{\prime}} \leqslant \chi_{\varkappa}(h) \leqslant C_{2} h^{\theta^{\prime}}
$$

а если, кроме того, выполняются вложения (35), то

$$
C_{3} h^{\theta^{\prime}} \leqslant \chi(h) \leqslant C_{4} h^{\theta^{\prime}}
$$

әде $C_{i}=C_{i}(\Omega, m, k, p, q, \varepsilon, \varkappa)>0$ при $i=1,2$ и $C_{i}=C_{i}(\Omega, m, k, p, q, \varepsilon)>0$ nрu $i=3,4$.

ДокаЗАТЕЛЬСТво. Возьмем множество $\Delta \subset \Omega \mathrm{c} \bar{h}(\Delta)=h, \underline{h}(\Delta)>\varkappa^{*} h$ (такое множество сушествует ввиду $\left.h<h_{2}\right)$ и шар $B\left(a, \varepsilon_{0} / 2\right) \subset \Omega_{(\varepsilon)}$. Далее $A_{i}=A_{i}(\Omega, m, k, p, q, \varepsilon)$ при условии (35) и $A_{i}=A_{i}(\Omega, m, k, p, q, \varepsilon, \varkappa)$ в противном случае; $A_{i}>0$. Очевидно, сушествуют натуральное число $t>A_{1} h^{-n}$ и попарно непересекаюшиеся шары $B\left(a_{i}, A_{2} h\right) \subset B\left(a, \varepsilon_{0} / 2\right) \backslash \Delta, i=\overline{1, t}$. Полагая $f=g\|g\|_{W_{p}^{k}(\Omega)}^{-1}$, где $g(x)=\varphi\left(\frac{x-a_{1}}{A_{2} h}\right)$ при $p \leqslant q$ и $g(x)=\sum_{i=1}^{t} \varphi\left(\frac{x-a_{i}}{A_{2} h}\right)$ при $p>q$, имеем

$$
\left\|D^{l}(f-s)\right\|_{L_{q}\left(\Omega_{(\varepsilon)}\right)}=\left\|D^{l} f\right\|_{L_{q}\left(\Omega_{(\varepsilon)}\right)} \geqslant A_{3} h^{\theta} .
$$

Из $(38)$ и из того, что топология факторпространства $W_{q}^{l}(B(0,1)) / \mathscr{P}_{l-1}$ порождается нормой $\left\|u+\mathscr{P}_{l-1}\right\|=\left\|D^{l} u\right\|_{L_{q}(B(0,1))}$ (лемма 3 ), вытекает, что если $g(x)=g\left(x_{1}, \ldots, x_{n}\right)=x_{1}^{2 m}$ и $\mathscr{L}^{m} v(x)=0$ на $B(0,1)$, то

$$
\left\|D^{l}(g-v)\right\|_{L_{q}(B(0,1))} \geqslant A_{4},
$$


а так как $\mathscr{L}^{m} s(x)=0$ на $\Omega \backslash \Delta$ (см. доказательство леммы 9$)$, для функции $f=$ $g\|g\|_{W_{p}^{k}(\Omega)}^{-1}$ получаем

$$
\left\|D^{l}(f-s)\right\|_{L_{q}\left(B\left(a_{i}, A_{2} h\right)\right)} \geqslant A_{5} h^{2 m-l+n / q} \quad \forall i=\overline{1, t}
$$

и, следовательно,

$$
\left\|D^{l}(f-s)\right\|_{L_{q}\left(\Omega_{(\varepsilon)}\right)} \geqslant A_{6} h^{2 m-l} .
$$

Верхние оценки при $k \leqslant 2 m$ вьполняются по теореме 2. Пусть $k>2 m$ и $r=$ $n(2 m-k+n / p)^{-1}$ при $k-n / p<2 m, r=q$ при $k-n / p \geqslant 2 m$. Тогда $r \geqslant 1, l<2 m$, $2 m-n / r \geqslant 2 m-n>l-n / q, 2 m-n / r \geqslant 2 m-n>m-n / 2,2 m-n / r \leqslant k-n / p$, причем если в последнем неравенстве имеет место равенство, то или $r<\infty$, или $p=1$ (так как $(k, p, q) \notin T)$. Отсюда согласно [38; $\S 9,10]$ следуют вложения $W_{r}^{2 m} \rightarrow W_{q}^{l}, W_{r}^{2 m} \rightarrow W_{2}^{m}$ и $W_{p}^{k} \rightarrow W_{r}^{2 m}$. По лемме 1 также $W_{p}^{k}(\Omega) \rightarrow W_{r}^{2 m}(\Omega)$. Кроме того, $(\theta(k, p)<2 m-l) \Leftrightarrow(r<q)$, так что $\theta(2 m, r)=\theta^{\prime}(k, p)$. Если $\bar{h}(\Delta)=h$ и верно либо (4), либо (35), то на основании сказанного и теоремы 2

$$
\left\|D^{l}(f-s)\right\|_{L_{q}\left(\Omega_{(\varepsilon)}\right)} \leqslant A_{7} h^{\theta(2 m, r)}\|f\|_{W_{r}^{2 m}(\Omega)} \leqslant A_{8} h^{\theta^{\prime}(k, p)}\|f\|_{W_{p}^{k}(\Omega)} .
$$

Таким образом, наилучший порядок приближения сплайнами в $W_{q}^{l}\left(\Omega_{(\varepsilon)}\right), \varepsilon>0$, которого можно добиться за счет достаточной гладкости интерполируемой функции, есть $O\left(\bar{h}^{2 m-l}\right)$. Сейчас допустим, что $\Omega-$ куб $(0,1)^{n}, W_{2}^{m} \rightarrow W_{q}^{l}$ и покажем, что порядок приближения в $W_{q}^{l}(\Omega)$ для любого класса $W_{p}^{k}(\Omega)$ интерполируемых функций не может быть вьше чем $O\left(\bar{h}^{m-l+1 / q}\right)$. Для этого положим $f(x)=f\left(x_{1}, \ldots, x_{n}\right)=x_{1}^{m}, \Delta=\Omega \cap\left(h \mathbb{Z}^{n}\right)$, где $h>0$ достаточно мало, $u\left(x_{1}\right)=\int_{0}^{1} \cdots \int_{0}^{1} s\left(x_{1}, \ldots, x_{n}\right) d x_{2} \cdots d x_{n}$ при $n>1$ и $u=s$ при $n=1$. Пусть $I=(0, h), v \in L_{2}(I), w$ - функция из $W_{2}^{m}(I)$, у которой $w^{(m)}=v$ на $I$ и $w^{(j)}(h)=0$ при $j=\overline{0, m-1}, g(x)=w\left(x_{1}\right)$ при $x_{1}<h$ и $g(x)=0$ при $x_{1} \geqslant h(x \in \Omega)$. По определению сплайна

$$
\int_{0}^{h} u^{(m)} v d x_{1}=\int_{\Omega} \sum_{|\alpha|_{1}=m} \frac{m !}{\alpha !} D^{\alpha} s D^{\alpha} g d x=0,
$$

так что $u$ совпадает с некоторым полиномом степени $<m$ на интервале $I$, следовательно,

$$
\left\|D^{l}(f-s)\right\|_{L_{q}(\Omega)} \geqslant\left\|\left(x_{1}^{m}-u\left(x_{1}\right)\right)^{(l)}\right\|_{L_{q}(I)} \geqslant A h^{m-l+1 / q}
$$

где $A=A(m)>0$.

ЗАмечАниЕ 4. Во всех полученных нами оценках погрешности сплайн-интерполяции мультипликативные константы зависят от величины $\varkappa$, если не наложено условие (35), которое является достаточным для выполнения оценок с константами, не зависящими от $\varkappa$. В работах автора [5], [6] получено необходимое для этого условие, близкое к достаточному условию (35), т.е. в соответствуюших случаях построены примеры расходимости интерполяционного процесса. ${ }^{7}$

\footnotetext{
${ }^{7}$ Результат, касающийся необходимых условий, недавно был несколько усилен А. Ю. Шадриньмм.
} 
Теперь рассмотрим приближение функций $f$, определенных на $\Omega$, сплайнами $s\left(f, \Delta, m, \mathbb{R}^{n}\right)$, которые благодаря их простой аналитической структуре (при конечном $\Delta)$ нашли широкое применение в решении разнообразных практических задач. Напомним, что сплайн $s\left(f, \Delta, m, \mathbb{R}^{n}\right)$ определяется как решение вариационной задачи.

ЛЕМма 11. При условиях теоремы 2

$$
\begin{aligned}
\| s(f, \Delta, m, \Omega)-s(f, \Delta, & \left.m, \mathbb{R}^{n}\right) \|_{W_{q}^{l}\left(\Omega_{(\varepsilon)}\right)} \\
& \leqslant C_{1} C_{2}^{1 / \bar{h}}\left(\omega_{2 m-k}\left(D^{k} f, \bar{h}\right)_{L_{p}(\Omega)}+\omega_{m}(f, \bar{h})_{L_{1}(\Omega)}\right),
\end{aligned}
$$

әде $C_{i}$ зависят от тех же параметров, что и в теореме $2,0<C_{2}<1$.

ДокАЗАтЕльство. Здесь будут использоваться обозначения $\varepsilon_{0}, \lambda, \tilde{f}, Z, V_{x}$, $V_{x}^{\prime}$, введенные в $\S 1$. Положим $\mu=\min \left\{1, \frac{\varepsilon}{6}, \varkappa\right\}$ в случае 1 ) теоремы 2 и $\mu=$ $\min \left\{1, \frac{\varepsilon}{6}\right\}$ в случае 2). Ниже $A_{i}=A_{i}(\Omega, m, p, q, \mu)>0$. Пусть $z \in Z, V_{z} \cap$ $\left(\lambda \Omega_{(\varepsilon)}\right) \neq \varnothing, F=\lambda \Omega^{(\varepsilon / 2)}$,

$$
\begin{gathered}
s_{1}=s(\tilde{f}, \lambda \Delta, m, \lambda \Omega), \quad s_{2}=s\left(\tilde{f}, \lambda \Delta, m, \mathbb{R}^{n}\right), \\
s_{3}=s\left(s_{1}-s_{2},(\lambda \Delta) \cup F, m, \lambda \Omega\right) .
\end{gathered}
$$

В случае 1$),$ поскольку $\lambda \underline{h} \geqslant 1$,

$$
\left\|D^{m} s_{1}\right\|_{L_{2}(\lambda \Omega)}+\left\|D^{m} s_{2}\right\|_{L_{2}\left(\mathbb{R}^{n}\right)} \leqslant A_{1}(\bar{h})^{-n} \max _{y \in \Delta}|f(y)|,
$$

а в случае 2) $f$ можно продолжить на $\mathbb{R}^{n}$ так, чтобы $\|f\|_{W_{2}^{m}\left(\mathbb{R}^{n}\right)} \leqslant A_{2}\|f\|_{W_{2}^{m}(\Omega)}$, см. [1] $\left(f \in W_{2}^{m}(\Omega)\right.$ по лемме 1$)$, так что

$$
\left\|D^{m} s_{1}\right\|_{L_{2}(\lambda \Omega)}+\left\|D^{m} s_{2}\right\|_{L_{2}\left(\mathbb{R}^{n}\right)} \leqslant A_{3}\|f\|_{W_{2}^{m}(\Omega)}
$$

Поэтому в обоих случаях

$$
\left\|D^{m} s_{3}\right\|_{L_{2}(\lambda \Omega)} \leqslant A_{4}\left(\left\|D^{m} s_{1}\right\|_{L_{2}(\lambda \Omega)}+\left\|D^{m} s_{2}\right\|_{L_{2}(\lambda \Omega)}\right) \leqslant A_{5}(\bar{h})^{-n}\|f\|_{W_{p}^{k}(\Omega)}
$$

(лемма 1). В силу леммы 6 ( $F$ определено выше, $\rho=\lambda \bar{h})$ и неравенства $\operatorname{dist}\left(F, V_{z}^{\prime}\right) \geqslant$ $A_{6}(\bar{h})^{-1}-A_{7}$ имеем

$$
\left\|s_{3}\right\|_{W_{2}^{m}\left(V_{z}^{\prime}\right)} \leqslant A_{8} A_{9}^{1 / \bar{h}}\left\|D^{m} s_{3}\right\|_{L_{2}(\lambda \Omega)}
$$

где $A_{9}<1$. Заметим, что $B(z, 2) \subset \lambda \Omega_{(\varepsilon / 2)}$, так как $\lambda \varepsilon / 2>3$, и, как легко видеть, $s_{3}=s_{1}-s_{2}$. Отсюда, из $(44),(45)$ и леммы 9 следует

$$
\left\|s_{1}-s_{2}\right\|_{W_{q}^{l}\left(V_{z}\right)} \leqslant A_{10}(\bar{h})^{-n} A_{9}^{1 / \bar{h}}\|f\|_{W_{p}^{k}(\Omega)} .
$$

Количество интересующих нас $z \in Z$ не превосходит $A_{11}(\bar{h})^{-n}$, поэтому, переходя к функциям на $\Omega$, получаем

$$
\left\|s(f, \Delta, m, \Omega)-s\left(f, \Delta, m, \mathbb{R}^{n}\right)\right\|_{W_{q}^{l}\left(\Omega_{(\varepsilon)}\right)} \leqslant A_{11} A_{12}^{1 / \bar{h}}\|f\|_{W_{p}^{k}(\Omega)},
$$


где $A_{12}<1$. Согласно леммам 4 (при $r=\lambda \operatorname{diam} \Omega>\lambda \varepsilon_{0}>1$ ) и 5 найдутся такие полиномы $P_{1} \in \mathscr{P}_{2 m-1}, P_{2} \in \mathscr{P}_{m-1}$, что

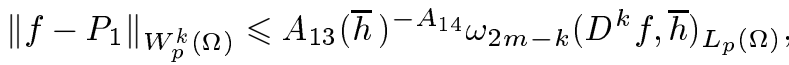

$$
\begin{aligned}
& \left\|f-P_{2}\right\|_{L_{1}(\Omega)} \leqslant A_{13}(\bar{h})^{-A_{14}} \omega_{m}(f, \bar{h})_{L_{1}(\Omega)},
\end{aligned}
$$

откуда

$$
\begin{aligned}
\left\|P_{1}-P_{2}\right\|_{W_{p}^{k}(\Omega)} & \leqslant A_{15}\left\|P_{1}-P_{2}\right\|_{L_{1}(\Omega)} \\
& \leqslant A_{16}(\bar{h})^{-A_{14}}\left(\omega_{2 m-k}\left(D^{k} f, \bar{h}\right)_{L_{p}(\Omega)}+\omega_{m}(f, \bar{h})_{L_{1}(\Omega)}\right) .
\end{aligned}
$$

Остается заметить, что в правой части (46) можно заменить $f$ на $f-P_{2}$, и оценить $\left\|f-P_{2}\right\|_{W_{p}^{k}(\Omega)}$ на основании (47), (48).

Из теоремы 2 и леммы 11 получаем

СлеДСТВИЕ 2. Теорема 2 останется справедливой, если заменить в ней $s=s(f, \Delta, m, \Omega)$ на $s\left(f, \Delta, m, \mathbb{R}^{n}\right)$.

Отметим, что в работе Ю. С. Завьялова, А. Имамова [43] и в книге В. А. Василенко [36] допущена ошибка: там утверждается, что в случае, когда $\Omega$ ограничено $и \Delta$ конечно, сплайн $s=s(f, \Delta, m, \Omega)$ может быть представлен в виде (7), где $\xi_{y} \in \mathbb{R} u P \in \mathscr{P}_{m-1}$. Допустим, что это так и $\Delta \subset \Omega$. Из определения сплайна вытекает сушествование таких $\xi_{y}^{\prime} \in \mathbb{R}(y \in \Delta)$, что

$$
\int_{\Omega} \sum_{|\alpha|_{1}=m} \frac{m !}{\alpha !} D^{\alpha} s D^{\alpha} g d x=\sum_{y \in \Delta} \xi_{y}^{\prime} g(y) \quad \forall g \in W_{2}^{m}(\Omega) .
$$

Поскольку $K(x-y)$ совпадает (с точностью до постоянного множителя) с фундаментальньм решением уравнения $\mathscr{L}^{m} u(x)=0$ (см. [41]),

$$
\int_{\Omega} \sum_{|\alpha|_{1}=m} \frac{m !}{\alpha !} D^{\alpha} s D^{\alpha} g d x=(-1)^{m} \int_{\Omega} s \cdot \mathscr{L}^{m} g d x=\sum_{y \in \Delta} C \xi_{y} g(y) \quad \forall g \in \mathscr{D}(\Omega),
$$

где $C=C(m, n) \neq 0$, так что $\xi_{y}^{\prime}=C \xi_{y}$; отсюда и из (49) следует

$$
\sum_{y \in \Delta} \xi_{y} Q(y)=0 \quad \forall Q \in \mathscr{P}_{m-1}
$$

Обозначим сплайн $s\left(f, \Delta, m, \mathbb{R}^{n}\right)$ через $\sigma$. Как показал Ж. Дюшон [28], $\sigma(x)$ имеет вид

$$
\sum_{y \in \Delta} \eta_{y} K(x-y)+\sum_{|\alpha|_{1}<m} c_{\alpha} x^{\alpha},
$$

где коэффициенты $\eta_{y}, c_{\alpha}$ удовлетворяют системе линейных уравнений

$$
\sigma(x)=f(x) \quad(x \in \Delta), \quad \sum_{y \in \Delta} \eta_{y} y^{\alpha}=0 \quad\left(|\alpha|_{1}<m\right) .
$$


Эта система, таким образом, имеет решение при любых значениях $f(x)$; в силу (9) система $\sum_{y \in \Delta} \eta_{y} y^{\alpha}=a_{\alpha}\left(|\alpha|_{1}<m\right)$ имеет решение при любых $a_{\alpha} \in \mathbb{R}$, поэтому, заменяя в $(50)$ нуль на $a_{\alpha}$, получим систему, которая имеет решение при любых $f(x), a_{\alpha}$. Следовательно, $\eta_{y}, c_{\alpha}$ однозначно определяются из уравнений $(50)$.

Итак, $s(x)=\sigma(x)$ на $\Omega$. Если $g(x)$ - некоторая функция из $L_{2}^{m}\left(\mathbb{R}^{n}\right)$, равная $\sigma(x)$ при $x \notin \Omega$ и равная нулю на $\Delta$, то по определению $s, \sigma$

$$
\begin{aligned}
\int_{\mathbb{R}^{n} \backslash \Omega} \sum_{|\alpha|_{1}=m} & \frac{m !}{\alpha !}\left(D^{\alpha} \sigma\right)^{2} d x \\
& =\int_{\mathbb{R}^{n}} \sum_{|\alpha|_{1}=m} \frac{m !}{\alpha !} D^{\alpha} \sigma D^{\alpha} g d x-\int_{\Omega} \sum_{|\alpha|_{1}=m} \frac{m !}{\alpha !} D^{\alpha} s D^{\alpha} g d x=0
\end{aligned}
$$

т.е. всегда $\sigma(x)$ совпадает с полиномом степени $<m$ на некотором шаре, а значит, ввиду свойства единственности полигармонических функций [44], и на всем $\mathbb{R}^{n}$, что, очевидно, неверно.

\section{§3. Аппроксимация сплайнами при $k=m, \theta=0$}

В этом параграфе мы снова возвращаемся к приближению сплайнами на всей области $\Omega$, которую будем предполагать ограниченной. Рассмотрим ситуацию, когда неравенство (10) ничего не говорит о сходимости $s\left(f, \Delta_{i}, m, \Omega\right)$ к $f$ при $\bar{h}\left(\Delta_{i}\right) \rightarrow 0$, т.е. когда $k=m$ и $\theta=0 .{ }^{8}$ Прежде всего выделяется "гильбертов" случай $k=l=m, p=q=2$. Здесь, в действительности, имеет место сходимость [28], [30], причем это единственный из известных случаев сходимости сплайнов в $W_{p}^{k}(\Omega)$ к функциям $f$ из того же класса $W_{p}^{k}(\Omega)$ без дополнительных условий на последовательность $\left\{\Delta_{i}\right\}$ при $n>1$. Это также исторически первьй результат по аппроксимации многомерными сплайнами. Однако, несмотря на простоту доказательства этого факта, которое основано непосредственно на вариационном принципе, из него (доказательства) нельзя извлечь какие-либо оценки сходимости через характеристики функции $f$; такие оценки не были известны и при $n=1$. Ниже дается оценка погрешности сплайн-интерполящии в указанной ситуации. Ввиду вложения $W_{p}^{k}(\Omega) \rightarrow W_{q}^{l}(\Omega)$ можно ограничиться случаем $k=l, p=q$.

Теорема 4. Допустим, что $k=m$, при $n>1$ nусть $p \leqslant 2$, nрu $n=1$ $\partial \Omega \subset \Delta$, а если $p \neq 2$, то выполняется (4). Тогда

$$
\left\|D^{k}(f-s)\right\|_{L_{p}(\Omega)} \leqslant C\left(\nu^{1 / p}\left\|D^{k} f\right\|_{L_{p}(\Omega)}+\left\|D^{k} f\right\|_{L_{p}(\Omega)}^{1-1 / p} \omega\left(D^{k} f, \nu\right)_{L_{p}(\Omega)}^{1 / p}\right),
$$

əде $\nu=\bar{h}|\ln \bar{h}|, C=C(\Omega, m, p, \varkappa)$ nрu $p \neq 2, C=C(\Omega, m)$ nри $p=2$.

То, что с ростом $р$ неравенство (51) становится хуже, вполне естественно, если судить по одномерному случаю: здесь при $p<\infty$ по теореме 4 есть сходимость в $W_{p}^{m}(\Omega)$ (для квазиравномерных последовательностей сеток, содержаших $\partial \Omega$ ), а при $p=\infty$ - нет, так как $s^{(m)}(x)=0$ в граничных точках $\Omega$ (если $m>1$ ), см. [23].

Доказательству теоремы предпошлем следующую лемму.

\footnotetext{
8 Заметим, что при условиях теоремы 2 всегда или $k<2 m$, или $\theta>0$.
} 
Лемма 12. Если $g \in L_{p}(\Omega) u r>0, m o$

$$
\|g\|_{L_{p}\left(\Omega^{(r)}\right)} \leqslant C\left(r^{1 / p}\|g\|_{L_{p}(\Omega)}+\|g\|_{L_{p}(\Omega)}^{1-1 / p} \omega(g, r)_{L_{p}(\Omega)}^{1 / p}\right)
$$

əде $C=C(\Omega)$.

ДокАЗАТЕльство. Заметим, что, во-первых, по лемме 2 область $\Omega$ является объединением конечного числа областей $\Omega_{j}, j \in J$, звездных относительно шара, а тогда $\Omega^{(r)} \subset \bigcup_{j \in J} \Omega_{j}^{(r)}$, и, во-вторых, как нетрудно проверить, ограниченная область, звездная относительно шара, имеет минимально гладкую границу. Поэтому лемму 12 достаточно доказать при дополнительном условии, что $\Omega$ звездна относительно шара $B(0,1)$, что мы и будем предполагать. Далее $A_{i}=A_{i}(\Omega)$.

Пусть $d=\operatorname{diam} \Omega, p<\infty, 0<r<\min \left\{\frac{1}{2}, \frac{\varepsilon_{0}}{2 d+1}\right\}$, где $\varepsilon_{0}-$ константа $C_{1}$ из леммы $2, a \in \mathbb{R}^{n}, U_{a}=B(a, r) \cap((1-r) \Omega) \neq \varnothing, V=\frac{1}{1-r} U_{a}, \varepsilon=(2 d+1) r$ и $G_{j}, j \in J,-$ области, удовлетворяющие условиям 1$\left.)-4\right)$ из леммы 2 . Поскольку $\Omega$ звездна относительно $B(0,1)$, имеем

$$
(1-r) \Omega \subset \Omega_{(r)} .
$$

Если $x \in B(a, r)$, то $|x|<(1-r) d+2 r \leqslant d$ (так как $d \geqslant 2)$ и $r<\frac{1}{2}$, откуда $\left|x-\frac{1}{1-r} x\right| \leqslant 2 d r$, так что $\frac{1}{1-r} B(a, r) \subset B(a,(2 d+1) r)$. Следовательно, $U_{a}, V \subset \Omega \cap B(a, \varepsilon)$, поэтому при некотором $j$

$$
U_{a}, V \subset G_{j} \subset \Omega \cap B\left(a, A_{1} r\right) .
$$

Положим $\mu=\frac{1}{\mathfrak{v} \varepsilon^{n}} \int_{B} g d x$, где $B$ - шар радиуса $\varepsilon$, относительно которого звездна данная область $G_{j}$. Ввиду (53)

$$
\begin{aligned}
\left\|g(x)-g\left(\frac{x}{1-r}\right)\right\|_{L_{p}\left(U_{a}\right)} \leqslant & \|g(x)-\mu\|_{L_{p}\left(U_{a}\right)}+\left\|g\left(\frac{x}{1-r}\right)-\mu\right\|_{L_{p}\left(U_{a}\right)} \\
= & \frac{1}{\mathfrak{v} \varepsilon^{n}}\left(\int_{U_{a}}\left|\int_{B}(g(x)-g(y)) d y\right|^{p} d x\right)^{1 / p} \\
& +\frac{(1-r)^{n / p}}{\mathfrak{v} \varepsilon^{n}}\left(\int_{V}\left|\int_{B}(g(x)-g(y)) d y\right|^{p} d x\right)^{1 / p} \\
\leqslant & A_{2} r^{-n / p}\|g(x)-g(y)\|_{L_{p}\left(Y_{a}\right)}
\end{aligned}
$$

где $Y_{a}$ - множество точек $(x, y) \in \mathbb{R}^{2 n}$ таких, что $[x, y] \subset \Omega \cap B\left(a, A_{1} r\right)$. Если неравенство между крайними выражениями в (54) возвести в степень $p$ и просуммировать по всем $a \in \frac{r}{n} \mathbb{Z}^{n}$ с $U_{a} \neq \varnothing$, получим

$$
\left\|g(x)-g\left(\frac{x}{1-r}\right)\right\|_{L_{p}((1-r) \Omega)} \leqslant A_{3} r^{-n / p}\left(\iint_{|x-y|<2 A_{1} r}|g(x)-g(y)|^{p} d x d y\right)^{1 / p},
$$


откуда (если сделать в интеграле замену $z=y-x$ и проинтегрировать сначала по $x$ ) следует, что

$$
\left\|g(x)-g\left(\frac{x}{1-r}\right)\right\|_{L_{p}((1-r) \Omega)} \leqslant A_{4} \omega(g, r)_{L_{p}(\Omega)} .
$$

Обозначим для краткости

$$
N_{1}=\|g\|_{L_{p}(\Omega)}, \quad N_{2}=\|g\|_{L_{p}((1-r) \Omega)}, \quad N_{3}=\|g\|_{L_{p}\left(\Omega^{(r)}\right)}, \quad \omega=\omega(g, r)_{L_{p}(\Omega)} .
$$

Тогда на основании (55) справедливо $\left|N_{2}-(1-r)^{n / p} N_{1}\right| \leqslant A_{4} \omega$, поэтому

$$
N_{1}-N_{2} \leqslant A_{5}\left(r N_{1}+\omega\right) \text {. }
$$

Используя неравенство

$$
\xi^{p}-\eta^{p} \leqslant p \xi^{p-1}(\xi-\eta)
$$

где $0 \leqslant \eta \leqslant \xi$, а также $(52),(56)$, получаем

$$
N_{3} \leqslant\left(N_{1}^{p}-N_{2}^{p}\right)^{1 / p} \leqslant p^{1 / p} N_{1}^{1-1 / p}\left(N_{1}-N_{2}\right)^{1 / p} \leqslant A_{6}\left(r^{1 / p} N_{1}+N_{1}^{1-1 / p} \omega^{1 / p}\right) .
$$

ДокАЗАТЕЛЬСтво теоремЫ 4 . Здесь $A_{i}=A_{i}(\Omega, m, p, \varkappa)$ при $p \neq 2$ и $A_{i}=$ $A_{i}(\Omega, m)$ при $p=2 ; A_{i}>0$. Пусть $\varepsilon>0$ и $p<\infty$ (случай $p=\infty, n=1$ содержится в замечании 2). По лемме 10 и замечанию 3

$$
\left\|D^{k}(f-s)\right\|_{L_{p}\left(\Omega_{(\varepsilon)}\right)} \leqslant A_{1}\left(\omega\left(D^{k} f, \bar{h}\right)_{L_{p}(\Omega)}+\varepsilon^{-A_{2}} A_{3}^{\varepsilon / \bar{h}}\left\|D^{k} f\right\|_{L_{p}(\Omega)}\right),
$$

где $A_{3}<1\left(\right.$ то, что $\omega_{m}(f, \bar{h})_{L_{1}(\Omega)}$ оценивается через $\left\|D^{m} f\right\|_{L_{p}(\Omega)}$, следует, например, из леммы 4). Согласно замечаниям $1,2\left(\Omega^{\prime}=\Omega^{(2 \varepsilon)}\right)$

$$
\left\|D^{k}(f-s)\right\|_{L_{p}\left(\Omega^{(2 \varepsilon)}\right)} \leqslant A_{4}\left(\left\|D^{k} f\right\|_{L_{p}\left(\Omega^{(3 \varepsilon)}\right)}+A_{5}^{\varepsilon / \bar{h}}\left\|D^{k} f\right\|_{L_{p}(\Omega)}\right)
$$

где $A_{5}<1$. Полагая $\varepsilon=\frac{A_{2}+1}{\left|\ln A_{6}\right|} \bar{h}|\ln \bar{h}|$, где $A_{6}=\max \left\{A_{3}, A_{5}\right\}$, и учитьвая, что $\bar{h}<\frac{1}{2}$, получаем отсюда

$$
\left\|D^{k}(f-s)\right\|_{L_{p}(\Omega)} \leqslant A_{7}\left(\bar{h}\left\|D^{k} f\right\|_{L_{p}(\Omega)}+\omega\left(D^{k} f, \bar{h}\right)_{L_{p}(\Omega)}+\left\|D^{k} f\right\|_{L_{p}\left(\Omega^{(3 \varepsilon)}\right)}\right) .
$$

Оценивая последнее слагаемое по лемме 12, получаем (51).

Из теорем 4 и 1 вытекает

СлеДСтвИЕ 3. Если $f \in W_{2}^{m}(\Omega), \Delta_{i} \subset \bar{\Omega}(i \in \mathbb{N}) u h_{i}=\bar{h}\left(\Delta_{i}\right) \rightarrow 0 n p u$ $i \rightarrow \infty$, mo

$$
\left\|f-s\left(f, \Delta_{i}, m, \Omega\right)\right\|_{W_{2}^{m}(\Omega)}^{2}=O\left(h_{i}\left|\ln h_{i}\right|+\omega\left(D^{m} f, h_{i}\left|\ln h_{i}\right|\right)_{L_{2}(\Omega)}\right), \quad i \rightarrow \infty .
$$




\section{Список литературы}

1. Стейн И. Сингулярные интегралы и дифференциалњные свойства функций. М.: Мир, 1973.

2. Atteia M. Existence et détermination des fonctions "spline" à plusieurs variables // C. R. Acad. Sci. Paris. Sér. A. 1966. V. 262. № 10. P. 575-578.

3. Матвеев O. В. Сплайн-интерполяция функций нескольких переменных и базисы в пространствах Соболева // Труды МИАН. 1992. Т. 198. С. 125-152.

4. Никольский С. М. Соболева пространство // Матем. энциклопедия. Т. 5. М.: Сов. энциклопедия, 1985. С. 56-58.

5. Матвеев О. В. Аппроксимативные свойства интерполяционных $D^{m}$-сплайнов // Докл. AH CCCP. 1991. T. 321. № 1. C. 14-18.

6. Матвеев О.В. Интерполирование $D^{m}$-сплайнами на хаотических сетках // Дис. . . докт. физ.-матем. наук. Екатеринбург: Ин-т матем. и мех. УрО РАН, 1994.

7. Walsh J. L., Ahlberg J. H., Nilson E. N. Best approximation properties of the spline fit // J. Math. Mech. 1962. V. 11. № 2. P. 225-234.

8. Ahlberg J.H., Nilson E. N. Convergence properties of the spline fit // J. Soc. Ind. Appl. Math. 1963. V. 11. № 1. P. 95-104.

9. Ahlberg J.H., Nilson E.N., Walsh J.L. Best approximation and convergence properties of higher-order spline approximations // J. Math. Mech. 1965. V. 14. № 2. P. 231-243.

10. Ahlberg J. H., Nilson E. N., Walsh J. L. Convergence properties of cubic splines // Notices Amer. Math. Soc. 1966. V. 13. № 1. P. 140.

11. Ahlberg J. H., Nilson E. N. Polynomial splines on the real line // J. Approx. Theory. 1970. V. 3. № 4. P. 398-409.

12. Sharma A., Meir A. Convergence of spline-functions // Notices Amer. Math. Soc. 1964. V. 11. № 7. P. 768 .

13. Sharma A., Meir A. Degree of approximation of spline interpolation // J. Math. Mech. 1966. V. 15. № 5. P. 759-767.

14. Nord S. Approximation properties of the spline fit // BIT. 1967. V. 7. № 2. P. 132-144.

15. De Boor C. Odd-degree spline interpolation at a biinfiniteknote sequence // Lecture Notes in Math. 1976. V. 556. P. 30-53.

16. Субботин Ю. Н. Приближение функций класса $W^{k} H_{\omega}^{p}$ сплайнами порядка $m$ // Докл. AH CCCP. 1970. T. 195. № 5. C. 1039-1041.

17. Субботин Ю. Н. Приближение "сплайн" -функциями и оценки поперечников // Труды МИАН. 1971. Т. 109. С. 35-60.

18. Субботин Ю. Н. Сплайн-аппроксимация // Труды Саратов. зимней школы (1982). Ч. 1. Саратов: Изд-во Саратов. ун-та, 1983. С. 81-90.

19. Lucas T. R. Error bounds for interpolating cubic splines under various end conditions // SIAM J. Numer. Anal. 1974. V. 11. № 3. P. 569-584.

20. Marsden $M$. Cubic spline interpolation of continuous functions // J. Approx. Theory. 1974. V. 10. № 2. P. 103-111.

21. Зматраков Н. Л. Сходимость интерполяционного процесса для параболических и кубических сплайнов // Труды МИАН. 1975. Т. 138. С. 71-93.

22. Шадрин $A$. Ю. О приближении функций интерполяционнњгми сплайнами, заданньми на неравномерных сетках // Матем. сб. 1990. Т. 181. №9. С. 1236-1255.

23. Алберг Джс., Нильсон Э., Уоли Дж. Теория сплайнов и ее приложения. М.: Мир, 1972.

24. Стечкин С. Б., Субботин Ю. Н. Сплайны в вычислительной математике. М.: Наука, 1976.

25. Завьялов Ю. С., Квасов Б.И., Мирошниченко В. Л. Методы сплайн-функций. М.: Наука, 1980.

26. Корнейчук Н. П. Точные константы в теории приближения. М.: Наука, 1987.

27. Farwig $R$. Rate of convergence of Shepard's global interpolation formula // Math. Comp. 1986. V. 46. № 174. P. 577-590.

28. Duchon $J$. Interpolation des fonctions de deux variables suivant le principe de la flexion des plaques minces // RAIRO Modél. Math. Anal. Numér. 1976. V. 10. № 12. P. 5-12. 
29. Duchon J. Sur l'erreur d'interpolation des fonctions de plusieurs variables par les $D^{m}$-splines // RAIRO Modél. Math. Anal. Numér. 1978. V. 12. № 4. P. 325-334.

30. Бежсаев A. Ю. $D^{m}$-сплайны в задачах приближения функций на хаотических сетках // Автореф. дис. ... канд. физ.-матем. наук. Новосибирск: ВЦ СО АН СССР, 1985.

31. Madych W. R., Nelson S. A. Polyharmonic cardinal splines // J. Approx. Theory. 1990. V. 60. № 2. P. 141-156.

32. Ciesielski Z., Figiel T. Spline bases in classical function spaces on compact $C^{\infty}$ manifolds. I // Studia Math. 1983. V. 76. № 1. P. 1-58; II // Studia Math. 1983. V. 76. № 2. P. 95-136.

33. Субботин Ю. Н. Приближение сплайнами и гладкие базисы в $C[0,2 \pi] / /$ Матем. заметки. 1972. Т. 12. № 1. С. 43-51.

34. Чисельский 3. Базисы и К-функционалы для пространств Соболева над компактньми многообразиями класса $C^{\infty} / /$ Труды МИАН. 1983. Т. 164. С. 197-203.

35. Franke R. Scattered data interpolation: tests of some methods // Math. Comp. 1982. V. 38. № 157. P. 181-200.

36. Василенко В. А. Сплайн-функции: теория, алгоритмы, программы. Новосибирск: Наука, 1983

37. Игнатов М.И., Певный А. Б. Сплайн-аппоксимация плавных поверхностей // Препринт. Сер. Науч. докл. / Коми фил. АН СССР. № 149. Ськтывкар: Коми филиал АН CCCP, 1986.

38. Бесов О.В., Ильин В. П., Никольский С. М. Интегральные представления функций и теоремы вложения. М.: Наука, 1975.

39. Брудный Ю. А. Многомерньй аналог одной теоремы Уитни // Матем. сб. 1970. Т. 82. № 2. C. $175-191$.

40. Данфорд Н., Швари Дж. Линейные операторы. Общая теория. М.: Наука, 1962.

41. Соболев С. Л. Введение в теорию кубатурных формул. М.: Наука, 1974.

42. Соболев С. Л. Некоторые применения функционального анализа в математической физике. М.: Наука, 1988.

43. Завьялов Ю. С., Имамов А. О вариационных задачах теории сплайнов // Матем. анализ и смежные вопросы математики. Новосибирск: Наука, 1978. С. 27-36.

44. Привалов И., Пчелин Б. К общей теории полигармонических функций // Матем. сб. 1937. T. 2. № 4 . C. $745-758$.

Институт математики и механики УрО РАН

E-mail: Oleg.Matveev@imm.uran.ru
Поступила в редакцию 30.01 .1997 и 15.04 .1998 